\title{
Capital humano y ciclo económico: la inscripción en educación superior en la Unión Europea en el período 2000-2018
}

Ignacio Duran ${ }^{1}$

\section{Resumen}

El presente trabajo analiza el comportamiento cíclico de la inscripción en educación superior de adultos jóvenes. Para esto se desarrolla un modelo de datos de panel para los países de la Unión Europea entre el año 2000 y el 2018. En primer lugar, se encuentran efectos estadísticamente significativos del ciclo económico en la inscripción, los cuales difieren según características sociodemográficas como edad y sexo. En concreto, para la inscripción de jóvenes de entre 20 y 30 años se observa tanto efectos pro-cíclicos del PBI per cápita como contra-cíclicos de la tasa de desempleo, aunque no para otros rangos etarios. Al analizar por separado la inscripción a diferentes programas, se encuentra que, en programas de ciclo corto, grado y maestría la inscripción femenina se ve más afectada que la masculina por variaciones en el PBI per cápita. Se observan además diferencias en el comportamiento de la inscripción según el nivel de PBI per cápita y profundidad financiera del país.

Palabras claves: desempleo, ciclos económicos, educación superior, capital humano

\begin{abstract}
The current study aims to analyze the cyclical behavior of enrollment in higher education of young adults. For this, a panel data is constructed for the countries of the European Union between 2000 and 2018. First, I find statistically significant effects of the business cycle on enrollment, which differ according to sociodemographic characteristics such as age and sex. In particular, for the enrollment of young people between the ages of 20 and 30, the results show both pro-cyclical effects of GDP per capita and counter-cyclical effects of the unemployment rate, although not for other age ranges. When separately analyzing enrollment in different types of programs, it is found that in short-cycle, bachelor's and master's degree programs, female enrollment is more affected than male enrollment by variations in GDP per capita. There are also differences in the behavior of enrollment according to the level of GDP per capita and financial depth of the country.
\end{abstract}

Keywords: unemployment, business cycles, higher education, human capital

JEL: E24, E32, I23, J24

DOI: https://doi.org/10.46553/ensayos.3.3.2021.p77-105

Fecha de recepción: 12/04/2021; Fecha de aceptación: 20/08/2021

${ }^{1}$ Facultad de Ciencias Económicas, Universidad Católica Argentina, Alicia M. de Justo 1400, (C1107AAZ) C.A.B.A, Argentina. Email: ignacio_duran@uca.edu.ar. El autor quisiera agradecer a Ann Mitchell por sus comentarios, sugerencias y guía durante todo el proceso de elaboración del documento. También se agradece a Bárbara Guerezta por sus comentarios relacionados al análisis econométrico. 


\section{Introducción}

En las primeras dos décadas del presente siglo tanto los costos de oportunidad de la educación como la capacidad de pago de los estudiantes y sus familias sufrieron importantes fluctuaciones cíclicas. El período que este trabajo comprende está marcado por la crisis financiera global del 2008 y la gran recesión que siguió. Las diferencias existentes entre los países de la Unión Europea, como pueden ser sus instituciones laborales, niveles de ingreso y profundidad financiera, entre otras características, llevaron a que el impacto de la crisis en los mercados laborales, financieros y en la actividad económica fuera heterogéneo a lo largo del bloque. En lo que se refiere a mercados laborales, sin embargo, puede observarse una dinámica que se repite en la gran mayoría de los países. El mayor golpe se concentró en los adultos jóvenes y en aquellos con menores niveles educativos. Por esta razón, durante una recesión parece razonable que los jóvenes busquen refugio en las universidades, siempre que sus ingresos lo permitan.

La literatura empírica relativa al comportamiento cíclico de la inversión en capital humano ha encontrado resultados diversos a la pregunta del efecto del ciclo económico en la acumulación de este tipo de capital. Si bien trabajos enfocados en universidades de los Estados Unidos como son Betts \& McFarland (1995), Dellas \& Sakellaris (2003), Bogan \& Wu (2018) y Foote \& Grosz (2020) encuentran que la inversión en capital humano sigue un patrón contra-cíclico, estudios como los de Sakellaris \& Spilimbergo (2000) y Schafmeister (2016), ambos enfocados en estudiantes internacionales, no obtuvieron los mismos resultados. Mientras que el primero afirma que el comportamiento cíclico de la inscripción universitaria depende del nivel de desarrollo del país de origen del estudiante, el segundo no logra obtener resultados significativos. En cada caso, la interpretación de dichos resultados se apoya en la importancia relativa de los determinantes de la inversión en capital humano, los cuales sufren fluctuaciones a lo largo del ciclo económico. En particular, los costos de oportunidad y la capacidad de pago.

El objetivo del presente trabajo es contribuir en la comprensión del comportamiento cíclico de la inversión en capital humano. Como se mencionó, buena parte de los estudios empíricos previos tendieron a enfocarse en estudiantes de universidades de los Estados Unidos por lo que el presente estudio busca contribuir a la discusión del tema examinando si los resultados obtenidos en estudios precedentes se repiten en otras geografías. Con esto en mente, las bases de datos publicadas por Eurostat son de gran utilidad al permitir estudiar el tema para un panel compuesto por una gran cantidad de países, para un período de tiempo suficientemente largo. El análisis empírico realizado en el presente trabajo aprovecha estas bases de datos para estudiar en base a datos a nivel agregado la relación entre la inscripción a programas de nivel terciario y el ciclo económico en 27 países miembros de la Unión Europea. En el documento se utilizarán los términos educación terciaria o educación superior de manera indistinta para hacer 
referencia a los niveles 5 a 8 de la clasificación ISCED 2011', lo cual incluye tanto programas de nivel terciario de ciclo corto, como de grado, maestría y doctorado. La información disponible permite realizar el análisis no solamente para el total de los estudiantes inscriptos en alguno de los programas mencionados, sino también de manera desagrada para distintos grupos sociodemográficos y para diferentes programas de estudio, permitiendo así un análisis más completo de la relación bajo estudio.

Con esto en mente, las conclusiones alcanzadas por Sakellaris \& Spilimbergo (2000) son el punto de partida para este trabajo al momento de plantear una hipótesis. Sostienen que en países de mayor desarrollo la inscripción universitaria (inversión en capital humano) es contra-cíclica mientras que en países menos desarrollados es pro-cíclica, y argumentan que esto se debe a diferencias en las instituciones laborales, los niveles de ingreso y, por tanto, a la capacidad de ahorro, y a la profundidad financiera, entre otros, los cuales permiten un mayor o menor aprovechamiento de la caída del costo de oportunidad de la educación durante las recesiones según el caso. La hipótesis que se pondrá a prueba es que la inscripción universitaria se ve afectada por el ciclo económico, y que este efecto depende de los niveles de ingreso y profundidad financiera del país en cuestión. Esto resulta de interés por las implicancias de que la hipótesis se compruebe. Si se observara que la inscripción universitaria es contra-cíclica en países de ingresos altos y pro-cíclica en los de ingresos bajos, podría interpretarse que durante las recesiones los estudiantes son conscientes de la caída en los costos de oportunidad, pero que en países de ingresos bajos la caída en la capacidad de pago actúa como limitante para aprovechar el menor costo de oportunidad. Esto se repetiría hacia el interior de los países. Familias de ingresos altos podrían seguir acumulando capital humano mientras familias de ingresos bajos no tienen como financiar dichas inversiones.

De ser así, el capital humano sería otra vía por la que las recesiones impactan negativamente en la equidad y desigualdad. De comprobarse que la profundidad financiera tiene un impacto en reducir la pro-ciclicidad de la inscripción universitaria se contaría con una herramienta adicional para minimizar los efectos negativos de las recesiones en términos de desigualdad. Ante la caída de los ingresos el sistema financiero proveería la liquidez necesaria para evitar que la capacidad de pago actúe como limitante. En tal caso, los gobiernos deberían implementar políticas que fomenten la profundización financiera y la creación de mercados de créditos estudiantiles que garanticen un mayor acceso a la educación, particularmente para familias de ingresos bajos. También se pondrán a prueba los resultados alcanzados por Bedard \& Herman (2008), donde se afirma que la inscripción masculina y femenina a programas de posgrado se ven afectadas de manera diferenciada por el ciclo económico.

\footnotetext{
${ }^{1}$ La Clasificación Estandarizada Internacional de Educación (ISCED por sus siglas en inglés) es la clasificación internacional de referencia para la identificación de programas educativos por niveles y campos. ISCED fue desarrollada por la UNESCO a mediados de la década de 1970, revisada por primera vez en 1997 y por segunda vez en 2011, en coordinación con organismos internacionales y expertos regionales. La revisión buscó reflejar los cambios ocurridos en los sistemas educativos. Mientras los programas terciarios se dividían en niveles 5 y 6 según la clasificación ISCED 1997, la revisión de ISCED 2011 implicó una desagregación del nivel 5 en tres niveles (5, 6 y 7), convirtiendo al nivel 6 de ISCED 1997 en el nivel 8 de ISCED 2011. Puede hallarse más información acerca de ISCED 2011 en: http://uis.unesco.org/sites/default/files/documents/internationalstandard-classification-of-education-isced-2011-en.pdf
} 
Con tal fin, el trabajo se divide de la siguiente manera. En la sección 2 se realiza una descripción de la evolución de las distintas variables de interés durante el período de estudio. En la sección 3 se introduce el modelo de capital humano en el cual se apoya el trabajo y se revisa la literatura empírica relativa a los efectos del ciclo económico en la inscripción universitaria. En la sección 4 se describe la metodología y bases de datos a utilizar. En la sección 5 se reportan los resultados de la estimación del modelo econométrico y, por último, en la sección 6 se realiza una conclusión.

\section{El ciclo económico y la inscripción universitaria en la Unión Europea}

La crisis financiera global significó un duro golpe a la actividad económica del conjunto de países que componen la Unión Europea. Esto se ilustra claramente en la Figura 1, donde se observa cómo, previo a la crisis, el bloque de países experimentaba tasas de crecimiento cercanas al $3 \%$. Aunque el crecimiento logró mantenerse en terreno positivo en el 2008, no ocurrió lo mismo en 2009, año en que la contracción del bloque fue superior al 4\%. El rebote experimentado en los años 2010 y 2011 sería seguido nuevamente por una importante desaceleración en 2012 y 2013 . El crecimiento solamente comenzaría a estabilizarse a partir del 2014. El resultado de estas fluctuaciones es que la tasa de crecimiento anual promedio para el conjunto de estas economías pasó del 2,3\% en el período 2002-2007 a 0\% en el 2008-2013. Algo similar ocurrió con la tasa de desempleo. El descenso observado entre los años 2004 y 2008 se convertiría en un marcado ascenso que alcanzaría su punto más alto en el período de estudio en 2013. No sería sino hasta 2018 que la tasa de desempleo retornaría al nivel observado en 2008.

Figura 1: Tasa de crecimiento real del PBI y tasa de desempleo para EU-28

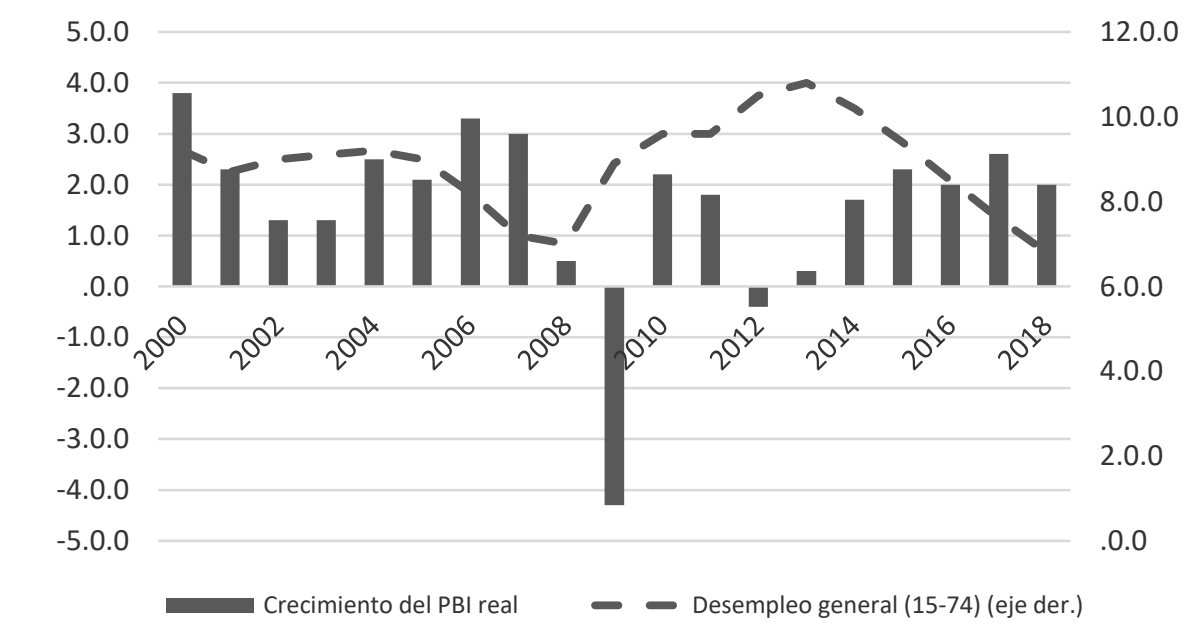

Fuente: Eurostat

Sin embargo, dicho golpe lejos estuvo de ser igual para todos los países de la unión. Mientras que el PBI se contrajo $14,8 \%$ en Lituania, $14,4 \%$ en Estonia y $14,2 \%$ en Letonia, países como Francia o Bélgica se vieron menos golpeados, con caídas del 
producto de $2,9 \%$ y $2 \%$, respectivamente. Polonia, por su parte, es el único país de la UE cuya economía no se contrajo a pesar de la crisis global.

Esta caída generalizada en la actividad económica se vio reflejada en los mercados laborales de los países de la Unión Europea de maneras diversas, en parte debido a las diferencias existentes en las instituciones laborales. Si bien en todos los países, a excepción de Alemania, la tasa de desempleo aumentó, la magnitud de dicho aumento varió enormemente.

Figura 2: Tasa de desempleo UE28 por grupos etarios

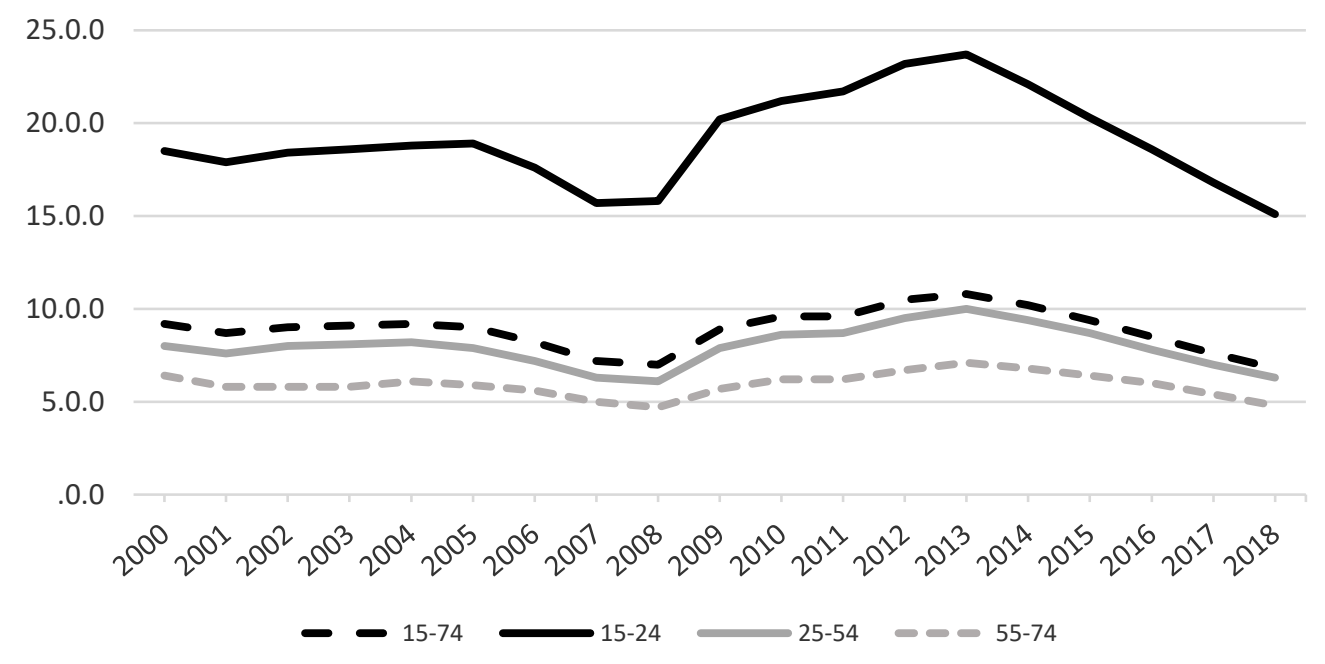

Fuente: Eurostat, Labour Force Survey

Sin embargo, como advierten Barakat et al. (2010) y OCDE (2012), el desempleo afectó a determinados grupos de maneras diferentes. En particular, esto es así para los más jóvenes y las personas con niveles educativos más bajos. Como puede verse en la Figura 2, entre el 2000 y 2018 la tasa de desempleo que enfrentaron los jóvenes europeos (de 15 a 24 años) no solo más que duplicó la tasa de desempleo general durante todo el periodo, sino que además sufrió fluctuaciones cíclicas significativamente superiores a las tasas de los otros grupos etarios. Esto se debe en parte a que las instituciones de los mercados laborales protegen proporcionalmente más a los trabajadores con mayor antigüedad. Además, en mercados laborales más rígidos, la caída en la demanda de trabajo afecta principalmente a quienes entran a la fuerza laboral, es decir, a los más jóvenes (Barakat et al., 2010).

También es verdad que los efectos del ciclo económico no lo sintieron de igual manera trabajadores con distintos niveles de instrucción. La Figura ilustra esto para la Unión Europea a partir del 2005 (primer año del que se tienen datos). Entre 2008 y 2013 la tasa de desempleo entre los trabajadores con título terciario (ISCED 5-8) aumentó de $3,8 \%$ a $6,4 \%$ mientras que para aquellos sin secundario completo el desempleo paso del $11,3 \%$ a casi el $20 \%$, para luego retornar hacia 2018 a niveles algo superiores a los anteriores a la crisis. La Figura 3 sugiere una mayor vulnerabilidad de los trabajadores 
con menores niveles educativos, los cuales, ante la recesión, se vieron en mayores dificultades para conseguir empleo que los trabajadores con estudios de nivel terciario.

Figura 3: Tasa de desempleo UE28 por nivel educativo alcanzado

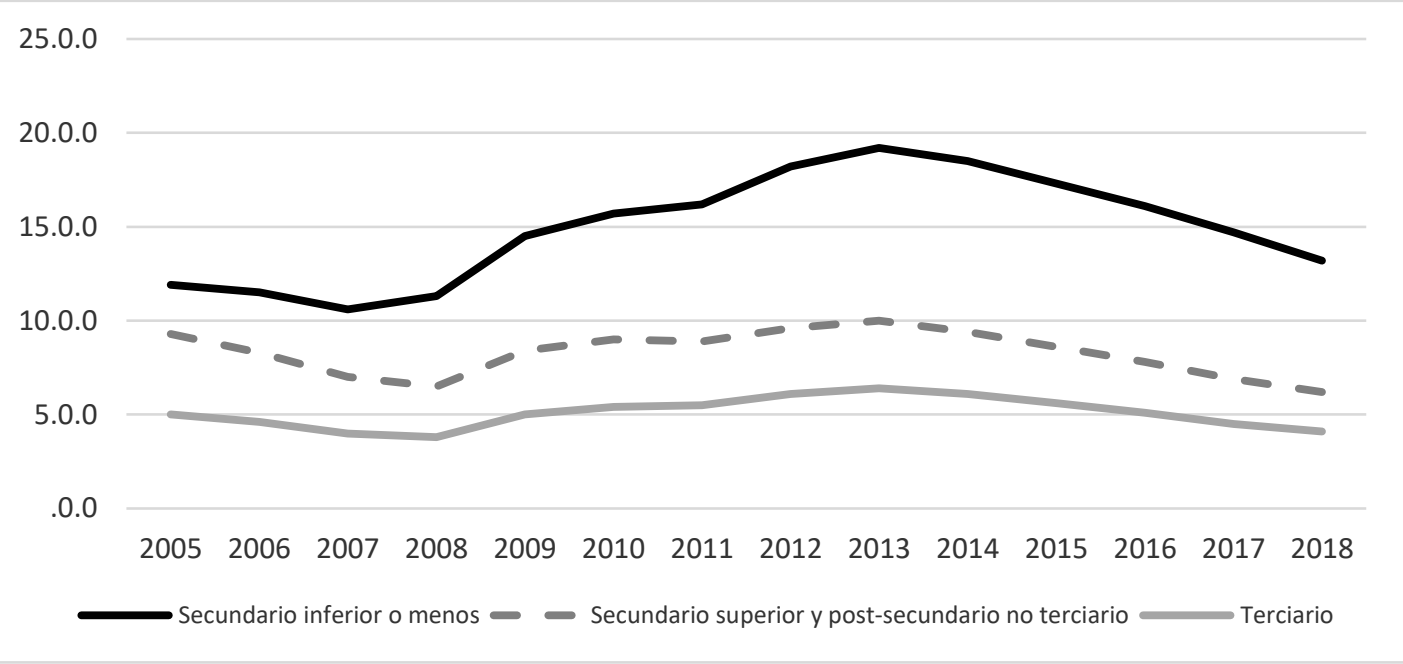

Fuente: Eurostat, Labour Force Survey

La pregunta a la que intenta responder este trabajo es qué efectos tuvo esto en la inscripción a educación superior en los distintos países de la Unión Europea. Trabajos como Karkkainen (2010) y Van Damme \& Karkkainen (2011), en base a datos preliminares obtenidos del Educationtoday Crisis Survey de la OCDE de junio de 2009 y junio de 2010, pronosticaban que el comportamiento general entre los países de la OCDE sería de aumento de la inscripción en educación superior. Skrbinjek et al. (2015) señalan que, si bien tanto en los países más fuertemente afectados por la crisis como en los menos afectados por ella la tasa bruta de inscripción en educación terciaria aumentó entre 2008 y 2012, dicho aumento fue superior en los países en que el impacto de la crisis en la economía fue mayor. En la misma línea, Ghita et al. (2013) muestran que en varios países de la Unión Europea los aumentos en las tasas de participación en educación superior continuaron a pesar la crisis económica, mientras que el abandono temprano de los estudios fue mayor previo a la crisis que luego de ésta.

\section{La inversión en capital humano y el ciclo económico}

\section{1. Marco teórico: el modelo de capital humano de Becker}

Considere la actividad $Y$. En dicha actividad los individuos reciben en el período en que se incorporan un salario $W_{Y, o}$. En el siguiente período los individuos reciben $W_{Y, 1}$, en el siguiente $W_{Y, 2}$, hasta $W_{Y, n}$ en el período que deciden retirarse. Se denota $W_{Y, t}$ al salario recibido en la actividad $Y$ para el período $t$. Incorporarse a dicha actividad, sin embargo, requiere realizar cierta inversión en capital humano, con un costo directo asociado $G y, t$. 
Los ingresos netos de la actividad $Y$ en el período $t$ resultan de sustraer $G_{Y, t}$ de $W_{Y, t}$ y se denotan con la letra $Y_{t}$. El valor presente de los ingresos netos de la actividad $Y$ sería:

$$
V(Y)=\sum_{t=0}^{n} \frac{Y_{t}}{(1+i)^{t+1}}
$$

Considere una segunda actividad $X$. La ganancia por elegir la actividad $Y$ sobre $X$ sería:

$$
d=V(Y)-V(X)=\sum_{t=0}^{n} \frac{Y_{t}-X_{t}}{(1+i)^{t+1}}
$$

Suponga ahora que la inversión que requiere la actividad $Y$ tiene una duración de cuatro períodos, y que impide trabajar por la duración de la misma. La actividad $X$, por otro lado, no requiere de inversión en capital humano. El costo de elegir la actividad $Y$ sobra $X$ es entonces la diferencia entre los ingresos netos en cada actividad durante los primeros cuatro períodos. El retorno de la inversión es la diferencia en los ingresos netos a partir del quinto período y hasta el período $n$. Denotando al retorno de la inversión en capital humano con la letra $R$ y al costo de ella $C$, la ecuación (2) puede reescribirse como:

$$
d=\sum_{t=4}^{n} \frac{Y_{t}-X_{t}}{(1+i)^{t+1}}-\sum_{t=0}^{3} \frac{X_{t}-Y_{t}}{(1+i)^{t+1}}=R-C
$$

Un individuo solamente estaría dispuesto a realizar la inversión que requiere incorporarse a la actividad $Y$ si ésta le reporta una ganancia, es decir, si $d>0$. La decisión de inversión en capital humano surge de la maximización del valor presente del retorno neto del valor presente de su costo. Dados los supuestos realizados, la ecuación (3) puede reescribirse de una manera que brinda aún más información:

$$
d=\sum_{t=4}^{n} \frac{W_{Y, t}-W_{X, t}}{(1+i)^{t+1}}-\sum_{t=0}^{3} \frac{W_{X, t}-G_{Y, t}}{(1+i)^{t+1}}=R-C
$$

Los costos de invertir en capital humano son los ingresos que debe resignar el individuo cuando elige la actividad $Y$, que requiere la inversión, en lugar de $X$ que no la requiere. Como puede observarse, estos no se limitan a los gastos directos $G y, t$ sino que incluyen los salarios $W_{x, t}$ que podría estar recibiendo si se dedicara a una actividad que no requiere inversión.

El supuesto de imposibilidad de trabajar por la duración de la inversión introduce la cuestión del financiamiento de esta inversión. Bajo el supuesto de mercados de crédito perfectos los individuos no tendrían problemas en conseguir financiamiento para inversiones de capital humano rentables. Sin embargo, bajo los supuestos más realistas 
de mercados imperfectos, Becker (1964) reconoce que la imposibilidad de establecer el capital adquirido como colateral y la dificultad en el cálculo de los retornos de la inversión (que generalmente se realizan temprano en la vida) actúan como limitantes a la hora de obtener un crédito para financiar este tipo de inversiones. Cuando esto ocurre el financiamiento debe provenir de los ahorros propios.

Los supuestos realizados no difieren tanto de la realidad que afronta un individuo al finalizar la escuela secundaria. En este caso $Y$ representaría realizar estudios universitarios mientras que $X$ insertarse directamente en el mercado laboral en un trabajo que requiera baja calificación. Aunque el supuesto de que $W_{y, t}=0$ para los cuatro primeros períodos (por la imposibilidad de trabajar durante el período de inversión) no siempre se cumple para los estudiantes universitarios, sí es verdad que los estudios universitarios reducen el tiempo que disponen los individuos para trabajar. También es verdad que, salvo por algunos países con mercados de créditos más desarrollados, el financiamiento de las inversiones en capital humano suele provenir de los ingresos familiares.

En el modelo desarrollado anteriormente, se dice que el costo de oportunidad de la inversión en capital humano es el salario $W_{x, t}$ que el individuo recibiría en caso de no realizar la inversión. Debe notarse que la alternativa a realizar estudios universitarios es ingresar a la fuerza laboral, lo cual no necesariamente implica estar empleado y recibiendo un salario. Es por eso que $W_{x, t}$ es realmente el salario estimado para dicha alternativa, el cual podría interpretarse como el salario efectivamente pagado en empleos de baja calificación multiplicado por la probabilidad de estar empleado. De esto surge que el costo de oportunidad de las inversiones de capital humano estaría determinado conjuntamente por el nivel de los salarios de entrada y la tasa de desempleo que afrontan los trabajadores de baja calificación.

En cuanto al comportamiento cíclico de las variables mencionadas anteriormente, mientras los salarios reales tienden a ser pro-cíclicos, las tasas de desempleo se comportan de manera contra-cíclica. Los costos de oportunidad, por tanto, parecen seguir un comportamiento pro-cíclico. Por el lado de los costos directos, es posible que medidas de austeridad durante una recesión impliquen una caída en las transferencias gubernamentales a las instituciones educativas, obligando a éstas a aumentar el valor de sus aranceles y costos de matriculación para compensar. Esto podría implicar un comportamiento contra-cíclico de los costos directos de la educación. Durante las recesiones, la caída del empleo y de los salarios reales afecta negativamente a la capacidad de pago. En el caso de crisis financieras, además, la caída del crédito puede restringir aún más la capacidad de los individuos de financiar la acumulación de capital humano.

En resumen, la decisión de inversión en capital humano depende de tres factores: los costos (directos y de oportunidad), el retorno de la educación, y la capacidad de pago. Mientras el comportamiento pro-cíclico del costo de oportunidad lleva a pensar que la inversión en capital humano aumentaría durante las recesiones, la capacidad de pagar por dicha inversión también cae durante las recesiones. Ya que no es posible predecir 
teóricamente que factor predomina, la literatura reciente respecto al tema se vio abocada a encontrar una respuesta empírica a la pregunta.

\section{2. Literatura empírica}

En este intento de dar una respuesta empírica a la pregunta acerca del comportamiento cíclico de la inversión en capital humano han surgido diversos enfoques en el abordaje de la cuestión. Mientras algunos autores basaron sus estudios en datos individuales, otros prefirieron utilizar datos agregados. Algunos autores se enfocaron en variables relacionadas al producto como indicadores del ciclo económico. Otros en las tasas de desempleo. Algunos estudiaron el comportamiento de la inscripción únicamente a carreras de grado, otros únicamente a carreras de posgrado. Y así como las metodologías variaron, también lo hicieron los resultados obtenidos.

Los estudios más relevantes basados en datos a nivel individuo se concentran principalmente en los Estados Unidos. Esto debido a la disponibilidad de bases de datos detalladas que incluyen características socioeconómicas de los individuos que podrían tener efectos en la inscripción universitaria. El trabajo realizado por Dellas \& Sakellaris (2003) se centra en la inscripción universitaria a nivel microeconómico valiéndose de los datos que surgen del Current Population Survey (CPS) del U.S. Bureau of Census. La muestra sobre la cual se realiza el estudio consiste en todos los graduados de secundaria de entre 18 y 22 años encuestados por el CPS entre 1968 y 1988. Desarrollan un modelo probit para la inscripción a la universidad controlando por características individuales (edad, género, raza y estado civil), utilizando para el ciclo económico las variables tasa de desempleo, diferencia del logaritmo de PNB real, y una medida del componente cíclico del producto construida por Cochrane (1994). Los resultados de este modelo, para cualquiera de las tres especificaciones de la variable de ciclo económico, parecen indicar comportamiento contra-cíclico de la inscripción universitaria. Según sus estimaciones el efecto marginal en la probabilidad promedio de inscripción a la universidad de un aumento de 1 punto porcentual en la tasa de desempleo sería un aumento de 0,8 puntos porcentuales. Para un aumento de 1 punto porcentual en la tasa de crecimiento del PNB el efecto sería una caída de 0,11 puntos porcentuales en la probabilidad de inscripción. $Y$, en tercer lugar, el efecto marginal de un valor de la medida de Cochrane de 0,01 (equivalente a un PNB 1\% por encima de su tendencia) sería una caída de 0,47 puntos porcentuales. El resultado se repite al replicar el modelo permitiendo la diferenciación entre inscripción a programas a tiempo parcial y a tiempo completo, aunque con mayor reacción por parte de la inscripción a tiempo completo a variaciones en la tasa de desempleo. Lo mismo ocurre para programas de dos y de cuatro años, siendo los programas de dos años algo más contra-cíclicos que los de 4 años. Por último, proceden a estimar un modelo más complejo en el que, además, de la tasa de desempleo incluyen otras variables que podrían afectar a la inscripción universitaria. Estiman que un aumento de 1 punto porcentual en la tasa de desempleo estatal está asociada a un aumento en la probabilidad de inscripción de 0,28 puntos porcentuales, un aumento de $\$ 40$ del salario semanal en manufacturas ( $\$ 1$ en salario por hora) se asocia a una caída en la probabilidad de inscripción de 0,8 puntos porcentuales, una suba de 1 punto porcentual en la tasa de interés real se asocia a una caída de 0,31 puntos porcentuales en la probabilidad de inscripción y un aumento de $1 \%$ en el diferencial de ingresos se 
asocia a un aumento en la probabilidad de inscripción de 0,32 puntos. Por otra parte, los costos de matriculación no parecen afectar significativamente la probabilidad de inscripción a la universidad. Concluyen así que el comportamiento contra-cíclico de la inscripción universitaria se explica esencialmente por una mayor reacción a los costos de oportunidad y de financiación que a los costos directos de la educación.

Mientras que Dellas \& Sakellaris (2003) utilizan datos a nivel individuos de los recién graduados del colegio secundario, el foco del trabajo presentado por Bedard \& Herman (2008) se encuentra en los recién graduados de carreras de grado en universidades de los Estados Unidos y los efectos del desempleo juvenil en la decisión entre ingresar a la fuerza laboral o continuar estudiando un título de posgrado. En base a datos del National Survey of Recent College Graduates (NSRCG) para el período 1993-2001 desarrollan modelos probit para la inscripción a doctorados, programas profesionales y maestrías (por separado) en los que las variables explicativas son la tasa de desempleo estatal, el GPA y el campo de estudio de la carrera de grado, controlando por características individuales como la edad, raza, estado civil, tiempo transcurrido desde la graduación, nacionalidad (utilizando variable dummy para nacidos en el extranjero), nivel educativo de la madre y cantidad de niños en el hogar en que vive. Encuentran que, para los hombres, un aumento de 1 punto porcentual en la tasa de desempleo aumenta la probabilidad de inscripción a doctorados en 0,15 puntos porcentuales, mientras que reduce la probabilidad de inscripción a maestrías en 0,58 puntos porcentuales. Los autores sugieren dos explicaciones para este comportamiento contra-cíclico de la inscripción masculina en doctorados y pro-cíclica en maestrías. La primera es que esto puede deberse a que, por ser más cortas, las maestrías actuarían como sustitutos de los doctorados en las etapas de expansión. La segunda es que, durante las recesiones, las maestrías a tiempo parcial financiadas por empleadores se ven reducidas. Este comportamiento cíclico, sin embargo, parece deberse a los individuos con GPA alto, mientras que no se ve un comportamiento cíclico en la inscripción de individuos de GPA bajo. En el caso de las mujeres tampoco es claro que la inscripción a ninguno de los tres programas tenga un comportamiento cíclico, tanto para GPAs altos o bajos.

Partiendo del hecho de que los ciclos económicos afectan de manera diferenciada a las minorías étnicas, Bogan \& Wu (2018) buscan identificar los efectos del ciclo económico en la inscripción a posgrados en los Estados Unidos para distintos grupos raciales. Para medir los efectos del ciclo económico en la inscripción utilizan un modelo probit en el que las variables explicativas relativas al ciclo económico son la tasa de desempleo y el nivel del índice S\&P 500, incluyendo hasta dos rezagos de las mismas, y controlando por características individuales como edad, genero, ingreso, y otras características socioeconómicas. Encuentran que un aumento de un punto porcentual en el desempleo incrementa la probabilidad de inscripción en 0,021 con un rezago de dos períodos. En maestrías un aumento de 1 punto en la tasa de desempleo incrementa probabilidad en 0,031 y en programas profesionales 0,015 pero reduce la probabilidad en doctorados en 0,019 . Al analizar lo que ocurre para los distintos grupos raciales encuentran que para los blancos los primeros dos rezagos del desempleo se asocian positiva y significativamente con la probabilidad de inscripción para maestrías y programas profesionales, indicando un comportamiento contra-cíclico. Para latinos y 
afroamericanos, por otra parte, no encuentran un comportamiento claramente pro ni contra-cíclico, debido a coeficientes de los rezagos con signos opuestos, o directamente no significativos.

Entre las investigaciones que abordaron la cuestión mediante datos a nivel agregado, uno de los primeros trabajos en cobrar relevancia fue el de Betts \& McFarland (1995). Este trabajo se enfoca en un sector en particular de la educación terciaria en los Estados Unidos: el de los community colleges ${ }^{2}$. Los autores sugieren que por las características particulares de estas instituciones y de los estudiantes inscriptos en ellas, los community colleges podrían funcionar como estabilizadores automáticos en momentos de recesión, y por tanto las inscripciones a ellos tendrían un comportamiento más fuertemente contra-cíclico que en las universidades. Utilizando las bases de datos de Higher Education General Information Survey/Integrated Postsecondary Education Data System ("HEGIS"/"IPEDS") del Departamento de Educación de los Estados Unidos, que reportan los datos a nivel de las instituciones educativas, para el periodo 1969-1985 emplean un modelo de datos de panel para poner a prueba su hipótesis. Comienzan por un modelo simple en el que la variable dependiente es el logaritmo natural del número de inscriptos en programas a tiempo completo y la variable explicativa el desempleo adulto ( 18 a 65 años), y encuentran que un aumento de $1 \%$ en la tasa de desempleo estaría asociado a un aumento de la inscripción de 8,8\%. Luego agregan una segunda variable explicativa, el ingreso per cápita, y obtienen resultados similares. El modelo completo incluye una gran cantidad de variables explicativas entre las que se encuentran tasas de desempleo y salarios para diversas edades y niveles educativos, retornos de la educación esperados, asistencia financiera y costos de matriculación, tanto para programas de dos años en community colleges como para programas universitarios de 4 años. Encuentran que salarios de entrada más altos reducen la inscripción mientras que costos de matriculación más elevados en programas universitarios de 4 años aumentan la inscripción en los programas de dos años que ofrecen los community colleges, lo cual sugiere que actúan en cierto modo como sustitutos. En base a los resultados obtenidos, los autores proponen que las políticas de mercado laboral debieran estar unidas a las políticas educativas para proveer de recursos a estas instituciones y así permitir que puedan satisfacer la demanda fuertemente contra-cíclica de educación en community colleges durante las recesiones.

Otro trabajo interesante que basa su análisis empírico en datos agregados es el de Sakellaris \& Spilimbergo (2000), aunque con un enfoque sensiblemente distinto al de Betts \& McFarland (1995), ya que centran su investigación en los estudiantes extranjeros inscriptos en universidades de los Estados Unidos intentando determinar cuál es el impacto que tiene el ciclo económico en el país de origen en dicha inscripción. Para esto desarrollan un modelo de datos de panel para la diferencia del logaritmo natural del número de estudiantes extranjeros inscriptos en universidades de EE.UU. (dato que

\footnotetext{
${ }^{2}$ Los community colleges en Estados Unidos son instituciones de educación terciaria generalmente públicos y que ofrecen programas de dos años. Estas instituciones dictan programas vocacionales y técnicos que permiten a los estudiantes una mejor inserción en el mercado laboral con un nivel de calificación media. También es común entre estudiantes que buscan reducir los costos totales de estudiar una carrera de grado de 4 años completar los primeros dos años en un community college para luego transferir los créditos a una universidad que ofrezca programas de 4 años, ya que éstas suelen ser más costosas.
} 
surge de la base de IIE) contra la diferencia del logaritmo natural del PBI per cápita del país de origen, incluyendo hasta cinco rezagos de esta variable y dos de la variable dependiente. Los datos utilizados abarcan el período 1959-1997 e incluyen 74 países de origen. Los resultados de este modelo sugieren una relación positiva y estadísticamente significativa entre el crecimiento del producto y el crecimiento de la inscripción universitaria (un comportamiento pro-cíclico de la inscripción universitaria). Los coeficientes para los primeros cinco rezagos son positivos y estadísticamente significativos, lo cual interpretan como reflejo de que la inscripción a cada año incluye varias cohortes, siendo las recién llegadas más sensibles a condiciones económicas recientes. Reportan una elasticidad de corto plazo de 0,18 y de largo plazo de 1,07. Reconocen, sin embargo, que los países de origen de estos estudiantes extranjeros conforman un grupo considerablemente heterogéneo.

Por esta razón proceden a estimar el mismo modelo para diferentes sub-muestras de países que comparten ciertas características. Los criterios utilizados son: la pertenencia del país de origen a la OCDE, el nivel de desigualdad (medido en base al coeficiente de Gini), el nivel de PBI per cápita al inicio del período y el nivel educativo de la población. Encuentran que, si bien entre países no pertenecientes a la OCDE el comportamiento de la inscripción continúa siendo pro-cíclico con una elasticidad de largo plazo cercana a la reportada para total de los países, la inscripción de estudiantes provenientes de países miembros de la OCDE sigue un patrón contra-cíclico. Los autores interpretan que esto se debe a que los mercados laborales de países de la OCDE permiten transiciones más rápidas de los estudios al trabajo haciendo que el costo de oportunidad sea más cíclico. También sugieren que la capacidad de pago en los países de la OCDE es menos cíclica por ser países de ingresos más altos, menos volátiles y distribuidos de manera menos desigual. Al re-estimar el modelo separando a los países en dos sub-muestras según el PBI per cápita al inicio del período encuentran que el comportamiento en ambos grupos es pro-cíclico y se asemeja bastante a los resultados obtenidos de la estimación para el total de países. Los resultados se repiten cuando el criterio de clasificación es la desigualdad de ingresos. Tanto en países con Gini altos como bajos la inscripción es procíclica.

Goh (2009) es otro trabajo que, al igual que Bedard \& Herman (2008), estudia los efectos del ciclo económico en la inscripción a programas de posgrado de universidades de los Estados Unidos, aprovechando las bases de datos de IPEDS (Integrated Postsecondary Education Data System) para analizar el período 1976-2005. Para esto desarrolla un modelo de datos de panel en el que cada grupo i hace referencia a una institución de educación superior. Las variables indicativas del ciclo económico utilizadas son la tasa de desempleo estatal, tasa de crecimiento del empleo, crecimiento del PBI, crecimiento del ingreso disponible y crecimiento del ingreso personal disponible. El estudio analiza por separado la inscripción a primer programa profesional (first-time first-professional incluye programas de odontología, derecho, medicina, farmacéutica, veterinaria, entre otros) del resto de programas de posgrado. Para los primeros programas profesionales encuentra que el comportamiento es contra-cíclico, con resultados consistentes para cuatro de las cinco variables indicativas del ciclo económico. Un aumento de un punto porcentual en la tasa de crecimiento del empleo se asocia a 
una caída de $4,09 \%$ en la inscripción, mientras que un incremento en un punto en el crecimiento del PBI se asocia a una caída de 2,04\% de la inscripción a este tipo de programas. Además, incrementos de un punto porcentual en la tasa de crecimiento del ingreso personal y el ingreso disponible estarían asociadas a reducciones de $1,68 \%$ y $1,30 \%$, respectivamente. La autora argumenta que, dado que el $90 \%$ de los inscriptos en este tipo de programas lo hacen en campos relacionados al derecho y la medicina, y la motivación principal es lograr un mayor atractivo en el mercado laboral, tiene sentido que se vean afectadas por el ciclo económico. Aunque también resaltan que es probable que la decisión sea más en el timing de la inversión en capital humano y no tanto en la decisión de realizarla o no. Al analizar los resultados para el resto de programas se encuentra mayor variabilidad en los resultados. Utilizando la tasa de desempleo y de crecimiento del empleo se observan comportamientos pro-cíclicos de la inscripción. Un incremento de 1 punto en la tasa de desempleo se asocia a una caída de 0,032\%, mientras que un incremento de un punto en el crecimiento del empleo se asocia a un incremento de $2,8 \%$ en la inscripción. Para el resto de las variables de ciclo económico encuentra comportamiento contra-cíclico. Incrementos de 1 punto porcentual en las tasas de crecimiento del PBI, ingreso personal e ingreso disponible se asocian a reducciones en la inscripción de $1,63 \%, 2,14 \%$ y 2,09\%, respectivamente. La autora atribuye esto a la heterogeneidad de programas dentro de la categoría "resto" y la variabilidad en las importancias relativas del costo de oportunidad y capacidad de pago para los inscriptos a estos programas. Concluye, por tanto, que el efecto del ciclo económico en la inscripción depende del tipo de programa del que se trate.

Schafmeister (2016), al igual que habían hecho Sakellaris \& Spilimbergo (2000), centra su investigación en los estudiantes extranjeros. Aunque no en un solo país, sino que, aprovechando la base de datos de la OCDE, trabaja con parejas de países, donde los receptores son 19 países pertenecientes a la OCDE y sus contrapartes son 163 países del mundo. Así, para 1999-2012, analiza cómo el crecimiento del PBI per cápita en el país de origen afecta a la tasa de crecimiento de la inscripción universitaria en países de la OCDE. La variable dependiente en su modelo es la tasa de crecimiento de la inscripción de estudiantes extranjeros en los distintos países de la OCDE según su país de origen, mientras la variable explicativa es la tasa de crecimiento del PBI en el país de origen, para la cual incluye la observación contemporánea además de 5 rezagos. Si bien los resultados de este primer modelo no parecen indicar la existencia de un comportamiento cíclico de la variable en cuestión, en base a los resultados de Sakellaris \& Spilimbergo (2000) afirma que los comportamientos probablemente difieran según el país de origen pertenezca a la OCDE o no, el nivel de ingreso y a desigualdad de ingresos. Sin embargo, aun corriendo la regresión por separado para las diferentes sub-muestras de países utilizando los criterios mencionados no logra extraer un comportamiento cíclico claro de la inscripción universitaria. Argumenta que esto se debe al alto grado de heterogeneidad entre los países.

Al igual que Betts \& McFarland (1995), Foote \& Grosz (2020) enfocan su estudio en la inscripción en community colleges en los Estados Unidos, analizando los efectos de los shocks en la demanda local de trabajo sobre la inscripción para el período 1996-2013. Para medir el tamaño del shock utilizan el número de trabajadores directamente afectado 
por un evento de despido masivo, el cual definen como por lo menos 50 trabajadores llenando pedidos de seguro de desempleo contra una misma institución en un período de cinco semanas. Los autores argumentan que al medir de esta manera los efectos del ciclo económico, se enfocan mejor en el mercado laboral local de las personas y los shocks son locales teniendo efectos más directos sobre los trabajadores, a diferencia de otras formas de medición que se enfocan en shocks a nivel macroeconómico. Para esto estiman un modelo de datos de panel en el que la variable independiente es la cantidad de personas directamente afectadas por eventos de despidos pasivos en el condado, incluyendo tres rezagos de la misma. Encuentran que un incremento de $1 \%$ en los despidos se asocia a una suba de $0,017 \%$ en los graduados en un período de tres años, siendo mayores los efectos en programas más cortos, lo cual se explica por mayor atrición en programas más largos. Además, estiman que, por cada 100 trabajadores afectados por eventos de despido masivo, la inscripción de primera vez en community colleges se incrementa en 2,8 estudiantes en un período de 3 años. Como puede observarse en la revisión bibliográfica realizada en esta sección, la cuestión del comportamiento cíclico de la inscripción universitaria no parece estar resuelta. Resultados heterogéneos y de difícil generalización dan lugar al presente trabajo.

\section{Metodología}

\section{IV.1. Modelo}

Como ya se dijo, el objetivo del trabajo es aportar a la discusión acerca del efecto del ciclo económico en la inversión en capital humano. Trabajos anteriores tendieron a enfocarse en la inscripción en universidades de los Estados Unidos, por lo que un trabajo que aborde la temática desde un panel de países puede aportar nueva información útil al debate. La metodología escogida busca contestar las tres preguntas planteadas al inicio del trabajo. En particular, si el ciclo económico afecta o no a la inversión en capital humano, si el efecto del ciclo económico en la inscripción difiere según edad y sexo, y si los niveles de ingreso o de profundidad financiera, en ambos casos a nivel de país, juegan algún rol en aquella relación.

El modelo que procederé a estimar es una adaptación del propuesto por Betts \& McFarland (1995). A diferencia de aquel, sin embargo, el presente estudio se enfoca en la inscripción terciaria en su conjunto en países de la Unión Europea. Si bien el estudio de una muestra compuesta por una mayor cantidad de países puede aportar información interesante acerca del comportamiento cíclico de la inscripción universitaria, esto es a costa de una menor disponibilidad de datos comparables entre los países (como podrían ser los costos de la educación, por ejemplo). Debido a que el principal objetivo del presente trabajo es avanzar en la comprensión del comportamiento cíclico de la inscripción universitaria de los jóvenes, y no hacer un estudio exhaustivo de los determinantes de la inscripción, el foco del modelo estará exclusivamente en las variables indicativas del ciclo económico utilizadas en los trabajos de Betts \& McFarland (1995) y Sakellaris \& Spilimbergo (2000) previamente citados: la tasa de desempleo y el logaritmo natural del PBI per cápita. Mientras que el desempleo, como se explica en 
la sección 3.1, es un determinante del costo de oportunidad de las inversiones de capital humano, el PBI per cápita actúa como indicador de la capacidad de pago de las familias. La ecuación del modelo sería:

$$
\operatorname{Ln}(E)_{i, t}=\alpha+\sum_{q=0}^{4} \beta_{q} U_{i, t-q}+\sum_{q=0}^{4} \gamma_{q} \operatorname{Ln}(P B I p c)_{i, t-q}+\xi_{i} * t+v_{i, t}
$$

La variable dependiente, $E_{i, t}$ es la cantidad total de estudiantes inscriptos en el país $i$ en el año $t$. La inscripción se incluye en logaritmos, misma transformación utilizada por Betts \& McFarland (1995), Sakellaris \& Spilimbergo (2000) y Schafmeister (2016). En la búsqueda de una mayor comprensión acerca del efecto del ciclo económico en la inscripción universitaria, se llevará a cabo el análisis de forma separada para distintos grupos sociodemográficos y programas de estudio, dada la disponibilidad de datos. En primer lugar, se analizará por separado la inscripción terciaria total (sin desagregar por tipo de programa) para los grupos etarios 18-19, 20-24, 25-29 y 30-34. Luego se procederá a estudiar la inscripción a programas de ciclo corto-grado-maestría por separado de la inscripción a programas de doctorado (en este caso sin desagregar por grupo etario). La imposibilidad de desagregar el primer grupo en sus tres componentes previo a 2013 es un limitante claro al análisis propuesto, ya que es evidente que las características de los estudiantes de programas de ciclo corto, grado y maestría difieren, y por tanto es lógico pensar que sus decisiones inversión en capital humano pueden verse afectadas de manera diferente por el ciclo económico. Sin embargo, estos programas también comparten características en común. Por lo general estos programas tienden a enfocarse en proveer a los participantes de conocimientos y habilidades profesionales, a diferencia de los programas de doctorado cuyo enfoque suele ser académico. Los programas también difieren en cuanto a su duración, siendo casi siempre los programas de grado y maestría más cortos que los de doctorado. La duración de los estudios es un factor importante si estos requieren que la persona se retire temporalmente del mercado laboral.

A priori, podría suponerse que la diferencia en el comportamiento de la inscripción de los estudiantes de diferentes edades está, al menos en parte, relacionada con los programas a los que se inscriben. Es mucho más probable que un estudiante menor de 24 años se encuentre estudiando su carrera de grado, o quizás una maestría, pero no tanto que estudie un doctorado (aunque no es imposible). Lo mismo al revés. Un estudiante mayor a 30 años probablemente estudie una maestría o un doctorado, y con menor probabilidad una carrera de grado. En todos los casos se analiza a la inscripción masculina y femenina en forma diferenciada. Esta metodología puede considerarse una adaptación de la utilizada por Bedard \& Herman (2008), donde se analizan por separado los efectos del ciclo económico en la inscripción masculina y femenina a distintos programas de posgrado, y se alcanzan resultados diferentes para cada caso. Analizar por separado la inscripción para los distintos programas de nivel terciario resulta necesario debido a las diferencias en las características de dichos programas y de sus estudiantes.

Las variables explicativas que se incluyen en el modelo son la tasa de desempleo (indicada en la ecuación como $U_{i, t}$ ) y el PBI per cápita, el cual no se incluye en niveles 
sino como logaritmo natural, además de incluir tendencias especificas por país, $\xi_{i} * t$. Como puede observarse en la ecuación, el modelo incluye además del valor contemporáneo de las variables independientes, cuatro rezagos de éstas para intentar identificar cualquier efecto en el tiempo que puedan tener sobre la inscripción terciaria. Para el desempleo se utilizan las tasas de desempleo anuales para distintos grupos etarios y para cada sexo, según corresponda para cada regresión. Los rangos de edades para los que se dispone información relativa a las tasas de desempleo y a la inscripción universitaria son coincidentes para tres de los cuatro grupos etarios bajo análisis: 2024, 25-29 y 30-34. De esta manera se evitan errores de estimación, los cuales no pueden evitarse para la inscripción del grupo 18-19, para el cual Eurostat no publica la tasa de desempleo. Para este grupo la tasa de desempleo utilizada es la del rango 15-19. Si bien el hecho de que los rangos de edades no coincidan perfectamente puede llevar a errores de medición, la importancia relativa de las cohortes 18-19 respecto de 15-17 en la fuerza laboral, lleva a asumir como válida la utilización de esta tasa. Estos posibles errores de medición son justamente la razón de que este grupo contenga un rango de edades menor a los tres restantes, dado que se priorizó el hecho de que los rangos de edades entre inscripción y tasa de desempleo fueran coincidentes siempre que fuera posible. Por otro lado, no tenía sentido dejar fuera del estudio al grupo 18-19 por su relevancia en la inscripción a programas de educación superior.

Al estimar el modelo para los distintos programas no se utilizan tasas de desempleo por edad sino por máximo grado académico alcanzado. De esta manera para la inscripción a doctorados se utiliza la tasa de desempleo para personas con estudios de terciario, mientras que, para los programas de ciclo corto, grado y maestría, se estimó en primer lugar el modelo utilizando la tasa de desempleo de personas con título de secundario superior y post-secundario no terciario, y luego nuevamente utilizando la tasa de desempleo de personas con título de nivel terciario. Es evidente que ninguna de estas dos refleja perfectamente la tasa de desempleo a la que se enfrentan los estudiantes de este grupo pudiendo derivar en errores de estimación, pero como se mencionó previamente, la disponibilidad de datos impide mayor desagregación. Para PBI per cápita los datos utilizados están expresados en euros constantes de 2010.

Conforme a los resultados de los tests realizados ${ }^{3}$ se determinó utilizar un modelo de Efectos Fijos, mediante la utilización de estimadores Within-Groups. Aunque esta decisión tiene sus fundamentos no solo en los resultados de los tests $F$ y de Hausman, sino también en los supuestos que deben hacerse sobre el efecto no observado. Dado que un modelo de Efectos Aleatorios requiere asumir que no existe correlación entre el efecto no observado $a_{i}$ y las variables explicativas $X_{i, t, j}$, se considera más sensato utilizar un modelo que sí permita la existencia de este tipo de correlación, como es el modelo de Efectos Fijos. Para sortear los posibles problemas de subestimación de errores estándar por autocorrelación de los errores, todas las regresiones se realizan con errores estándar agrupados por países.

\footnotetext{
${ }^{3}$ Se realizó el test de Levin-Lin-Chu (LLC) rechazando la presencia de raíces unitarias. También se realizaron tests $\mathrm{F}$ y Hausman, los cuales indicaron la preferencia de un modelo de Efectos Fijos por uno Pooled o de Efectos Aleatorios.
} 
No se pasa por alto el posible problema de endogeneidad por causalidad inversa que la elección de este modelo puede conllevar, producto de que mientras que el desempleo afecta a la inscripción universitaria, a su vez la inscripción afecta a la tasa de desempleo. Para evitar este tipo de problemas suelen usarse Modelos de Ecuaciones Simultaneas. En el presente estudio, sin embargo, se optó por no utilizar este modelo por problemas que surgen a la hora de estimarlo. Para que este modelo tenga sentido, cada una de las ecuaciones estructurales del sistema debe tener una interpretación ceteris paribus en aislamiento de la otra ecuación. Por esta razón es común que se utilice para casos en que se busca estimar oferta y demanda de un bien. En este caso, sin embargo, ambas variables endógenas son determinadas por una decisión de un mismo individuo, inscribirse en un programa universitario (retirándose de la fuerza laboral) o mantenerse desempleado.

\section{IV.2. Bases de datos}

Para el análisis empírico, los datos se obtuvieron de cuatro bases de datos de Eurostat, la oficina estadística de la Unión Europea. Las dos primeras sirven como fuente de datos relativos a la variable dependiente, la inscripción universitaria. Los datos utilizados para el estudio de esta variable surgen de las bases Education administrative data until 2012 (ISCED1997) y Education administrative data from 2013 onwards (ISCED 2011) compiladas por Eurostat. Esta información es recolectada a través de cuestionarios gracias al trabajo conjunto de UIS (UNESCO Institute of Statistics), OECD y Eurostat (UOE), y provista por los ministerios de educación u oficinas estadísticas según el país. La recopilación de datos de UOE sobre estadísticas de educación cubre los estados miembros de la UE (Bélgica, Bulgaria, Croacia, República Checa, Dinamarca, Alemania, Estonia, Grecia, España, Francia, Irlanda, Italia, Chipre, Letonia, Lituania, Luxemburgo, Malta, Hungría, Países Bajos, Austria, Polonia, Portugal, Rumania, Eslovenia, República Eslovaca, Finlandia, Suecia y Reino Unido), los países de la EFTA/EEA (Islandia, Liechtenstein, Noruega y Suiza), los países candidatos (Albania, Macedonia del Norte, Serbia y Turquía) y los estados miembros de la OCDE situados fuera de Europa (Australia, Canadá, Japón, Corea, México, Nueva Zelanda, Estados Unidos) y otros países (por ejemplo, Israel). Los datos cubren principalmente el período 1998 en adelante, siendo 2018 el último dato disponible al momento de escribir este trabajo.

Para el presente trabajo la muestra utilizada se compone únicamente de los datos relativos a los países miembros de la Unión Europea. Teniendo en cuenta que existieron incorporaciones al bloque en los años 2004 y 2013, se entiende en este trabajo por Unión Europea a los 28 países miembros entre 2013 y 2020. Muy pocos de estos 28 países reportan datos de inscripción a cursos de nivel terciario para los años 1998 y 1999. Por esto, el período estudiado va desde el año 2000 al 2018 . Solo existe un país para el que se observan demasiados valores faltantes y por tanto debe ser excluido del estudio: Luxemburgo. Así de este modo, se estudia una muestra compuesta por 27 países y que abarca un período de 19 años. El panel, sin embargo, es desbalanceado, ya que se observan valores faltantes. Esto podría generar estimadores sesgados en caso de que la razón por la que se da la atrición este correlacionada con el error idiosincrático, lo cual en ocasiones ocurre cuando se trata de individuos o firmas, por ejemplo. En este caso, por tratarse de países se asume que la razón de los valores faltantes no 
correlaciona con los errores y por tanto la estimación mediante un modelo de Efectos Fijos no generaría estimadores sesgados. Como se puede ver en la tabla a continuación, además, se observan valores mínimos considerablemente bajos para la inscripción en programas de doctorado. Dado que esto se da principalmente en Malta, el país es excluido de la muestra al momento de regresar el modelo para la inscripción a programas de doctorado.

De las dos bases restantes se obtuvieron los datos relativos al desempleo y el PBI per cápita. En el primer caso, la base de datos utilizada es LFS main indicators la cual contiene los datos de los principales indicadores del mercado laboral. La información contenida en dicha base surge del European Labour Force Survey (EU-LFS), la encuesta de hogares más grande de Europa, con información para los 28 países de la UE, 4 candidatos a unirse al bloque y 3 países pertenecientes a EFTA. Para el PBI per cápita, la información se extrae de la base de datos Annual National Accounts. Si bien es publicada por Eurostat, esta información es producida por las oficinas estadísticas de los respectivos países.

En la Tabla 1 se presentan estadísticas descriptivas de las variables que se utilizan en el análisis econométrico. Se observa que la media de inscripción femenina es superior a la masculina para todas las especificaciones de la variable, a excepción de la inscripción a doctorados, donde la inscripción masculina es algo superior. Lo mismo ocurre para las tasas de desempleo, donde la media para las mujeres supera a la de los hombres para ambos rangos etarios.

\section{Resultados}

En la Tabla 2 se reportan los resultados obtenidos de regresar el modelo para los distintos rangos etarios para el total de los estudiantes inscriptos en programas de educación superior, sin distinción por sexo. Como se mencionó, el rango etario de la tasa de desempleo coincide con el de la inscripción para todos los grupos a excepción de 1819, para el cual se utiliza la tasa de desempleo del grupo 15-19 como variable explicativa. 
Tabla 1: Resumen de las variables de estudio

\begin{tabular}{|c|c|c|c|c|c|}
\hline & \multicolumn{4}{|c|}{ Inscripción } & Máximo \\
\hline 18-19 & 509 & 106.503 & 154.189 & 557 & 846.314 \\
\hline Masculina & 509 & 45.569 & 69.040 & 197 & 395.791 \\
\hline Femenina & 509 & 60.933 & 85.531 & 360 & 450.523 \\
\hline $20-24$ & 508 & 343.676 & 397.595 & 3.238 & \#\#\#\#\#\# \\
\hline Masculina & 508 & 155.014 & 180.464 & 1.425 & 707.498 \\
\hline Femenina & 508 & 188.662 & 217.777 & 1.761 & 752.974 \\
\hline 25-29 & 508 & 125.795 & 167.018 & 622 & 949.282 \\
\hline Masculina & 508 & 62.419 & 89.066 & 307 & 523.808 \\
\hline Femenina & 508 & 63.376 & 79.198 & 315 & 425.473 \\
\hline $30-34$ & 505 & 54.039 & 69.191 & 161 & 356.968 \\
\hline Masculina & 505 & 25.316 & 34.881 & 95 & 208.107 \\
\hline Femenina & 505 & 28.723 & 35.873 & 66 & 148.861 \\
\hline Ciclo Corto/Grado/Maestría & 493 & 662.357 & 771.303 & 7.251 & \#\#\#\#\#\# \\
\hline Masculina & 493 & 296.438 & 349.485 & 3.123 & 1.586 .668 \\
\hline Femenina & 493 & 365.919 & 424.381 & 4.064 & 1.507 .781 \\
\hline Doctorado & 493 & 23.809 & 34.593 & 8 & 214.700 \\
\hline Masculina & 493 & 12.548 & 19.176 & 5 & 128.000 \\
\hline Femenina & 493 & 11.261 & 15.540 & 3 & 89.500 \\
\hline \multicolumn{6}{|l|}{ Tasa de Desempleo } \\
\hline 15-19 & 583 & 30.1 & 15.0 & 6.2 & 74.0 \\
\hline Masculina & 549 & 29.7 & 15.3 & 5.3 & 74.9 \\
\hline Femenina & 528 & 31.7 & 16.2 & 6.2 & 80.8 \\
\hline $15-24$ & 593 & 20.2 & 9.8 & 4.4 & 58.3 \\
\hline Masculina & 592 & 20.0 & 9.5 & 4.2 & 56.2 \\
\hline Femenina & 591 & 20.4 & 10.7 & 4.5 & 63.8 \\
\hline $20-24$ & 592 & 17.9 & 9.3 & 2.8 & 56.1 \\
\hline Masculina & 584 & 18.0 & 9.0 & 2.7 & 52.7 \\
\hline Femenina & 582 & 18.1 & 10.3 & 3.0 & 61.2 \\
\hline $25-29$ & 588 & 11.0 & 6.0 & 2.0 & 43.3 \\
\hline Masculina & 580 & 10.6 & 5.7 & 1.3 & 40.8 \\
\hline Femenina & 574 & 11.7 & 6.8 & 2.2 & 46.3 \\
\hline $30-34$ & 588 & 8.5 & 4.5 & 1.4 & 30.2 \\
\hline Masculina & 572 & 7.8 & 4.4 & 1.0 & 28.0 \\
\hline Femenina & 573 & 9.5 & 5.2 & 1.6 & 34.6 \\
\hline Secundario Superior & 586 & 9.2 & 4.9 & 1.7 & 31.2 \\
\hline Masculina & 583 & 8.5 & 4.6 & 1.4 & 26.4 \\
\hline Femenina & 583 & 10.1 & 5.7 & 2.0 & 37.0 \\
\hline Terciario & 582 & 5.0 & 2.9 & 1.2 & 20.4 \\
\hline Masculina & 558 & 4.6 & 2.5 & 1.0 & 17.0 \\
\hline Femenina & 570 & 5.5 & 3.6 & 1.0 & 24.5 \\
\hline PBI per cápita & 611 & 21.553 & 11.917 & 2.860 & $\mathbf{5 7 . 7 8 0}$ \\
\hline
\end{tabular}

Fuente: elaboración propia. 
Tabla 2: Inscripción universitaria por edad

\begin{tabular}{|c|c|c|c|c|c|}
\hline & & & Tota & & \\
\hline & 18 a 19 & 20 a 24 & & 25 a 29 & 30 a 34 \\
\hline Constante & $\begin{array}{l}54.035 \\
(20.733)\end{array}$ & $\begin{array}{r}54.621 \\
(7.346)\end{array}$ & $* * *$ & $\begin{array}{l}26.325 \text { ** } \\
(12.146)\end{array}$ & $\begin{array}{c}68.285 * \\
(36.022)\end{array}$ \\
\hline $\begin{array}{l}\text { Desempleo } \\
(\mathrm{t})\end{array}$ & $\begin{array}{r}-0.003 \\
(0.007)\end{array}$ & $\begin{array}{r}0.006 \\
(0.002)\end{array}$ & $* * *$ & $\begin{array}{r}0.009 \\
(0.005)\end{array}$ & $\begin{array}{r}0.005 \\
(0.013)\end{array}$ \\
\hline$(t-1)$ & $\begin{array}{r}-0.001 \\
(0.002)\end{array}$ & $\begin{array}{r}0.001 \\
(0.001)\end{array}$ & & $\begin{array}{r}-0.004 \\
(0.003)\end{array}$ & $\begin{array}{r}-0.006 \\
(0.007)\end{array}$ \\
\hline$(\mathrm{t}-2)$ & $\begin{array}{r}0.001 \\
(0.001)\end{array}$ & $\begin{array}{r}0.000 \\
(0.001)\end{array}$ & & $\begin{array}{r}-0.006 \\
(0.005)\end{array}$ & $\begin{array}{r}-0.011 \\
(0.009)\end{array}$ \\
\hline$(\mathrm{t}-3)$ & $\begin{array}{r}0.003 \\
(0.002)\end{array}$ & $\begin{array}{r}0.001 \\
(0.001)\end{array}$ & & $\begin{array}{r}0.003 \\
(0.003)\end{array}$ & $\begin{array}{r}0.000 \\
(0.005)\end{array}$ \\
\hline$(t-4)$ & $\begin{array}{r}0.004 \\
(0.003)\end{array}$ & $\begin{array}{r}0.007 \\
(0.002)\end{array}$ & $* * *$ & $\begin{array}{r}-0.002 \\
(0.003)\end{array}$ & $\begin{array}{r}0.009 \\
(0.010)\end{array}$ \\
\hline $\begin{array}{l}\text { Ln(PBI per cápi } \\
(\mathrm{t})\end{array}$ & $\begin{array}{r}0.384 \\
(0.348)\end{array}$ & $\begin{array}{r}0.610 \\
(0.153)\end{array}$ & $* * *$ & $\begin{array}{r}0.458 \\
(0.416)\end{array}$ & $\begin{array}{r}1.822 \\
(1.114)\end{array}$ \\
\hline$(t-1)$ & $\begin{array}{r}0.166 \\
(0.393)\end{array}$ & $\begin{array}{r}0.245 \\
(0.109)\end{array}$ & $* *$ & $\begin{array}{r}0.285 \\
(0.257)\end{array}$ & $\begin{array}{r}-0.091 \\
(0.582)\end{array}$ \\
\hline$(t-2)$ & $\begin{array}{r}-0.268 \\
(0.381)\end{array}$ & $\begin{array}{r}0.170 \\
(0.097)\end{array}$ & * & $\begin{array}{r}0.057 \\
(0.175)\end{array}$ & $\begin{array}{r}-0.112 \\
(0.288)\end{array}$ \\
\hline$(t-3)$ & $\begin{array}{r}0.594 \\
(0.603)\end{array}$ & $\begin{array}{r}0.245 \\
(0.105)\end{array}$ & $* *$ & $\begin{array}{r}-0.021 \\
(0.243)\end{array}$ & $\begin{array}{r}0.262 \\
(0.367)\end{array}$ \\
\hline$(t-4)$ & $\begin{array}{r}0.584 \\
(0.500) \\
\end{array}$ & $\begin{array}{r}0.380 \\
(0.206) \\
\end{array}$ & $*$ & $\begin{array}{r}0.358 \\
(0.394) \\
\end{array}$ & $\begin{array}{r}0.723 \\
(0.565) \\
\end{array}$ \\
\hline Observaciones & 451 & 476 & & 472 & 469 \\
\hline Grupos & 27 & 27 & & 27 & 27 \\
\hline$R^{2}$ & 0.008 & 0.035 & & 0.000 & 0.000 \\
\hline Within & 0.471 & 0.819 & & 0.688 & 0.593 \\
\hline Between & 0.023 & 0.018 & & 0.010 & 0.000 \\
\hline
\end{tabular}

A simple vista se observa que los efectos del ciclo económico en la inscripción en programas de educación superior parecen diferir según la edad de los estudiantes. Mejor dicho, en esta regresión inicial, solo se observan efectos del ciclo económico en las inscripciones de personas de entre 20 y 24 años. No solo eso, además se obtienen coeficientes estadísticamente significativos para ambas variables indicativas del ciclo económico. Además, en ambos casos los coeficientes son de signo positivo, lo cual podría interpretarse como evidencia de la presencia de factores tanto pro-cíclicos como contracíclicos. Al momento de describir el modelo de capital humano, se mencionó que el ciclo económico afectaría a este tipo de inversiones por dos vías: el costo de oportunidad y la capacidad de pago. Mientras que el efecto del costo de oportunidad induciría a un comportamiento contra-cíclico de la inscripción, el efecto de la capacidad de pago llevaría a un comportamiento pro-cíclico de ésta. Por un lado, un aumento en un punto porcentual en la tasa de desempleo se asocia a un incremento en la inscripción de 0,6\% en el mismo período, suponiendo todo lo demás constante. Por otro, un incremento de $1 \%$ en el PBI per cápita también se ve asociado a un incremento en $0,61 \%$ en la 
inscripción del mismo período. En este segundo caso, sin embargo, se observan además efectos rezagados. El mismo aumento de $1 \%$ en el PBI per cápita supondría un incremento de $0,24 \%$ adicional en la inscripción en el período subsiguiente, y a incrementos de $0,17 \%, 0,24 \%$ y $0,38 \%$ con dos, tres y cuatro períodos de rezago, respectivamente.

Tabla 3: Inscripción universitaria por edad y sexo

\begin{tabular}{|c|c|c|c|c|c|c|c|c|}
\hline & \multicolumn{4}{|c|}{ Hombres } & \multicolumn{4}{|c|}{ Mujeres } \\
\hline & 18 a 19 & 20 a 24 & 25 a 29 & 30 a 34 & 18 a 19 & 20 a 24 & 25 a 29 & 30 a 34 \\
\hline Constante & $\begin{array}{r}23.652 \\
(26.674)\end{array}$ & $\begin{array}{l}46.518 * * * \\
(7.490)\end{array}$ & $\begin{array}{l}40.606 * * * \\
(7.834)\end{array}$ & $\begin{array}{l}94.573 * * * \\
(26.889)\end{array}$ & $\begin{array}{l}67.535 * * \\
(24.772)\end{array}$ & $\begin{array}{l}42.712 * * * \\
(9.323)\end{array}$ & $\begin{array}{l}29.747 * * * \\
(8.923)\end{array}$ & $\begin{array}{l}88.529 \text { *** } \\
(23.613)\end{array}$ \\
\hline $\begin{array}{l}\text { Desempleo } \\
(\mathrm{t})\end{array}$ & $\begin{array}{r}-0.006 \\
(0.008)\end{array}$ & $\begin{array}{l}0.005 * * \\
(0.002)\end{array}$ & $\begin{array}{r}0.005 \\
(0.005)\end{array}$ & $\begin{array}{r}0.007 \\
(0.012)\end{array}$ & $\begin{array}{r}-0.004 \\
(0.007)\end{array}$ & $\begin{array}{l}0.005 * * \\
(0.002)\end{array}$ & $\begin{array}{l}0.011 * * \\
(0.004)\end{array}$ & $\begin{array}{r}0.013 \\
(0.010)\end{array}$ \\
\hline$(t-1)$ & $\begin{array}{r}0.000 \\
(0.002)\end{array}$ & $\begin{array}{r}0.002 \\
(0.001)\end{array}$ & $\begin{array}{r}-0.001 \\
(0.004)\end{array}$ & $\begin{array}{r}0.000 \\
(0.006)\end{array}$ & $\begin{array}{r}-0.001 \\
(0.002)\end{array}$ & $\begin{array}{r}0.002 \\
(0.001)\end{array}$ & $\begin{array}{r}0.000 \\
(0.002)\end{array}$ & $\begin{array}{r}-0.002 \\
(0.009)\end{array}$ \\
\hline$(t-2)$ & $\begin{array}{r}0.001 \\
(0.003)\end{array}$ & $\begin{array}{r}0.001 \\
(0.001)\end{array}$ & $\begin{array}{r}-0.004 \\
(0.003)\end{array}$ & $\begin{array}{r}0.003 \\
(0.006)\end{array}$ & $\begin{array}{r}0.000 \\
(0.002)\end{array}$ & $\begin{array}{r}0.001 \\
(0.001)\end{array}$ & $\begin{array}{r}-0.006 \\
(0.006)\end{array}$ & $\begin{array}{r}-0.004 \\
(0.012)\end{array}$ \\
\hline$(t-3)$ & $\begin{array}{r}0.002 \\
(0.003)\end{array}$ & $\begin{array}{r}0.002 \\
(0.001)\end{array}$ & $\begin{array}{c}0.004 * * \\
(0.002)\end{array}$ & $\begin{array}{r}0.000 \\
(0.005)\end{array}$ & $\begin{array}{c}0.005 * \\
(0.003)\end{array}$ & $\begin{array}{r}0.000 \\
(0.001)\end{array}$ & $\begin{array}{r}0.001 \\
(0.004)\end{array}$ & $\begin{array}{r}-0.003 \\
(0.007)\end{array}$ \\
\hline$(t-4)$ & $\begin{array}{r}0.004 \\
(0.003)\end{array}$ & $\begin{array}{l}0.006 \text { *** } \\
(0.001)\end{array}$ & $\begin{array}{r}0.001 \\
(0.002)\end{array}$ & $\begin{array}{r}0.009 \\
(0.008)\end{array}$ & $\begin{array}{r}0.008 \\
(0.005)\end{array}$ & $\begin{array}{l}0.007 * * * \\
(0.002)\end{array}$ & $\begin{array}{r}-0.002 \\
(0.004)\end{array}$ & $\begin{array}{r}0.013 \\
(0.010)\end{array}$ \\
\hline $\begin{array}{l}\text { Ln(PBI per cáp } \\
(t)\end{array}$ & $\begin{array}{r}0.090 \\
(0.438)\end{array}$ & $\begin{array}{l}0.591 \text { *** } \\
(0.168)\end{array}$ & $\begin{array}{r}0.471 \\
(0.531)\end{array}$ & $\begin{array}{r}2.193 \\
(1.453)\end{array}$ & $\begin{array}{r}0.534 \\
(0.479)\end{array}$ & $\begin{array}{l}0.412 \text { ** } \\
(0.149)\end{array}$ & $\begin{array}{r}0.607 \\
(0.493)\end{array}$ & $\begin{array}{l}2.538 * \\
(1.295)\end{array}$ \\
\hline$(t-1)$ & $\begin{array}{r}0.140 \\
(0.472)\end{array}$ & $\begin{array}{r}0.148 \\
(0.126)\end{array}$ & $\begin{array}{r}0.433 \\
(0.260)\end{array}$ & $\begin{array}{r}0.226 \\
(0.536)\end{array}$ & $\begin{array}{r}-0.039 \\
(0.377)\end{array}$ & $\begin{array}{l}0.229 * \\
(0.128)\end{array}$ & $\begin{array}{r}0.501 \\
(0.343)\end{array}$ & $\begin{array}{r}0.042 \\
(0.753)\end{array}$ \\
\hline$(t-2)$ & $\begin{array}{r}-0.434 \\
(0.356)\end{array}$ & $\begin{array}{r}0.147 \\
(0.134)\end{array}$ & $\begin{array}{r}0.176 \\
(0.203)\end{array}$ & $\begin{array}{r}0.215 \\
(0.232)\end{array}$ & $\begin{array}{r}-0.297 \\
(0.472)\end{array}$ & $\begin{array}{r}0.226 \\
(0.134)\end{array}$ & $\begin{array}{l}0.580 * * * \\
(0.195)\end{array}$ & $\begin{array}{r}0.407 \\
(0.308)\end{array}$ \\
\hline$(t-3)$ & $\begin{array}{r}0.284 \\
(0.519)\end{array}$ & $\begin{array}{r}0.162 \\
(0.098)\end{array}$ & $\begin{array}{r}0.033 \\
(0.249)\end{array}$ & $\begin{array}{r}0.443 \\
(0.407)\end{array}$ & $\begin{array}{r}0.795 \\
(0.550)\end{array}$ & $\begin{array}{r}0.141 \\
(0.134)\end{array}$ & $\begin{array}{r}-0.083 \\
(0.263)\end{array}$ & $\begin{array}{r}0.664 \\
(0.447)\end{array}$ \\
\hline$(t-4)$ & $\begin{array}{r}0.724 \\
(0.644) \\
\end{array}$ & $\begin{array}{r}0.347 \\
(0.208) \\
\end{array}$ & $\begin{array}{r}0.558 \\
(0.424) \\
\end{array}$ & $\begin{array}{r}0.796 \\
(0.685) \\
\end{array}$ & $\begin{array}{r}0.714 \\
(0.681) \\
\end{array}$ & $\begin{array}{l}0.415 \text { ** } \\
(0.179)\end{array}$ & $\begin{array}{r}0.198 \\
(0.377) \\
\end{array}$ & $\begin{array}{r}0.769 \\
(0.634) \\
\end{array}$ \\
\hline Observaciones & 401 & 456 & 452 & 435 & 389 & 456 & 445 & 435 \\
\hline Grupos & 25 & 27 & 27 & 27 & 25 & 27 & 27 & 26 \\
\hline $\mathrm{R}^{2}$ & 0.000 & 0.0327 & 0.004 & 0.0347 & 0.004 & 0.023 & 0.003 & 0.005 \\
\hline Within & 0.533 & 0.8117 & 0.626 & 0.5487 & 0.402 & 0.823 & 0.775 & 0.655 \\
\hline Between & 0.035 & 0.0293 & 0.000 & 0.0384 & 0.010 & 0.021 & 0.005 & 0.004 \\
\hline
\end{tabular}

Notas: errores estándar entre paréntesis. Significatividad al $10 \%, 5 \%$ y $1 \%$ : *,**, ***, respectivamente.

El siguiente paso es analizar si la inscripción masculina y femenina se ven afectadas de igual manera por el ciclo económico. Luego de analizar la inscripción a programas de posgrados en los Estados Unidos, Bedard \& Herman (2008) concluyen que los efectos del ciclo económico en la inscripción a posgrados difieren según el sexo. En la Tabla 3 se reportan los resultados de regresar el mismo modelo que en la Tabla 2, solo que en este caso por separado según el sexo. Llama la atención en este caso lo que ocurre con la inscripción del grupo 25-29. En particular, puede observarse que un aumento de un punto en la tasa de desempleo se asocia a un incremento de 1,16\% en la inscripción de mujeres de entre 25 y 29 años en el mismo período, más del doble de lo que se observa para estudiantes de entre 20 y 24, para ambos sexos. Puede interpretarse esto como una mayor sensibilidad en las mujeres a cambios en los costos de oportunidad de la inversión en capital humano. Por su parte, en la inscripción femenina del grupo 30-34 sobresale el efecto contemporáneo del PBI per cápita en la inscripción, considerando que un aumento de $1 \%$ en dicha variable explicativa se asocia a un incremento de $2,54 \%$, 
evidenciando un comportamiento marcadamente pro-cíclico. En este sentido, si bien los resultados coinciden con los de Bedard \& Herman (2008) en el sentido de que la inscripción femenina y masculina parecen verse afectados de manera diferente por el ciclo económico, a diferencia de aquel, en el presente trabajo se observan importantes efectos del ciclo económico en la inscripción femenina, incluso mayores que en la masculina.

Tabla 4: Inscripción universitaria por programa y sexo

\begin{tabular}{|c|c|c|c|c|c|c|}
\hline & Grado/Maestría (1) & $\begin{array}{c}\text { Hombres } \\
\text { Grado/Maestría(2) }\end{array}$ & Doctorado & Grado/Maestría (1) & $\begin{array}{c}\text { Mujeres } \\
\text { Grado/Maestría (2) }\end{array}$ & Doctorado \\
\hline Constante & $\begin{array}{l}40.022 \text { *** } \\
(9.573)\end{array}$ & $\begin{array}{l}41.836^{* * *} \\
(7.795)\end{array}$ & $\begin{array}{r}7.127 \\
(14.520)\end{array}$ & $\begin{array}{l}4^{46.818^{* * *}} \\
(8.17)\end{array}$ & $\begin{array}{l}45.031^{* * *} \\
(9.20)\end{array}$ & $\begin{array}{c}1.046 \\
(13.29)\end{array}$ \\
\hline $\begin{array}{l}\text { Desempleo } \\
(\mathrm{t})\end{array}$ & $\begin{array}{c}0.009^{* *} \\
(0.005)\end{array}$ & $\begin{array}{c}0.016^{* *} \\
(0.006)\end{array}$ & $\begin{array}{r}-0.019 \\
(0.024)\end{array}$ & $\begin{array}{l}0.013^{* * *} \\
(0.005)\end{array}$ & $\begin{array}{c}0.013^{* *} \\
(0.006)\end{array}$ & $\begin{array}{l}-0.017 \\
(0.025)\end{array}$ \\
\hline$(t-1)$ & $\begin{array}{r}0.002 \\
(0.002)\end{array}$ & $\begin{array}{r}0.004 \\
(0.003)\end{array}$ & $\begin{array}{r}-0.001 \\
(0.011)\end{array}$ & $\begin{array}{r}0.002 \\
(0.002)\end{array}$ & $\begin{array}{r}0.007 \\
(0.005)\end{array}$ & $\begin{array}{r}-0.003 \\
(0.008)\end{array}$ \\
\hline$(t-2)$ & $\begin{array}{r}0.000 \\
(0.002)\end{array}$ & $\begin{array}{r}0.005 \\
(0.004)\end{array}$ & $\begin{array}{r}-0.020 \\
(0.012)\end{array}$ & $\begin{array}{r}-0.003 \\
(0.002)\end{array}$ & $\begin{array}{r}0.002 \\
(0.003)\end{array}$ & $\begin{array}{l}-0.005 \\
(0.008)\end{array}$ \\
\hline$(t-3)$ & $\begin{array}{r}0.001 \\
(0.002)\end{array}$ & $\begin{array}{r}0.005 \\
(0.003)\end{array}$ & $\begin{array}{l}0.020^{* *} \\
(0.008)\end{array}$ & $\begin{array}{r}-0.001 \\
(0.003)\end{array}$ & $\begin{array}{r}0.004 \\
(0.002)\end{array}$ & $\begin{array}{l}0.020^{* * *} \\
(0.007)\end{array}$ \\
\hline$(t-4)$ & $\begin{array}{c}0.007 \\
(0.004)\end{array}$ & $\begin{array}{l}0.012 \text { ** } \\
(0.005)\end{array}$ & $\begin{array}{r}0.021 \\
(0.018)\end{array}$ & $\begin{array}{r}0.009 \\
(0.004)\end{array}$ & $\begin{array}{r}0.006 \\
(0.005)\end{array}$ & $\begin{array}{r}0.026 \\
(0.017)\end{array}$ \\
\hline $\begin{array}{l}\text { Ln(PBI per cápit } \\
(t)\end{array}$ & $\begin{array}{l}0.6000^{* * *} \\
(0.180)\end{array}$ & $\begin{array}{l}0.547^{* * *} \\
(0.174)\end{array}$ & $\begin{array}{r}-0.147 \\
(0.504)\end{array}$ & $\begin{array}{l}0.7499^{* * *} \\
(0.195)\end{array}$ & $\begin{array}{l}0.596^{* * *} \\
(0.214)\end{array}$ & $\begin{array}{l}-0.270 \\
(0.410)\end{array}$ \\
\hline$(t-1)$ & $\begin{array}{c}0.303^{*} \\
(0.162)\end{array}$ & $\begin{array}{c}0.304^{*} \\
(0.154)\end{array}$ & $\begin{array}{r}-0.383 \\
(0.574)\end{array}$ & $\begin{array}{c}0.334^{* *} \\
(0.161)\end{array}$ & $\begin{array}{c}0.307^{* *} \\
(0.132)\end{array}$ & $\begin{array}{r}-0.294 \\
(0.399)\end{array}$ \\
\hline$(t-2)$ & $\begin{array}{r}0.009 \\
(0.152)\end{array}$ & $\begin{array}{c}0.190 \\
(0.110)\end{array}$ & $\begin{array}{r}0.277 \\
(0.291)\end{array}$ & $\begin{array}{r}0.172 \\
(0.104)\end{array}$ & $\begin{array}{c}0.266 \text { * } \\
(0.142)\end{array}$ & $\begin{array}{c}0.590 \\
(0.287)\end{array}$ \\
\hline$(t-3)$ & $\begin{array}{r}0.159 \\
(0.125)\end{array}$ & $\begin{array}{r}0.165 \\
(0.140)\end{array}$ & $\begin{array}{r}-0.205 \\
(0.355)\end{array}$ & $\begin{array}{r}0.141 \\
(0.104)\end{array}$ & $\begin{array}{c}0.344^{* *} \\
(0.127)\end{array}$ & $\begin{array}{r}0.066 \\
(0.325)\end{array}$ \\
\hline$(t-4)$ & $\begin{array}{r}0.236 \\
(0.232) \\
\end{array}$ & $\begin{array}{r}0.233 \\
(0.199) \\
\end{array}$ & $\begin{array}{c}1.304 \text { * } \\
(0.662)\end{array}$ & $\begin{array}{r}0.260 \\
(0.225) \\
\end{array}$ & $\begin{array}{r}0.104 \\
(0.195) \\
\end{array}$ & $\begin{array}{c}1.457^{* *} \\
(0.603) \\
\end{array}$ \\
\hline $\begin{array}{l}\text { Observaciones } \\
\text { Grupos }\end{array}$ & $\begin{array}{r}451 \\
27\end{array}$ & $\begin{array}{r}412 \\
26\end{array}$ & $\begin{array}{r}412 \\
26\end{array}$ & $\begin{array}{r}451 \\
27\end{array}$ & $\begin{array}{r}425 \\
27\end{array}$ & $\begin{array}{r}421 \\
26\end{array}$ \\
\hline $\mathrm{R}^{2}$ Within & $\begin{array}{l}0.033 \\
0.836\end{array}$ & $\begin{array}{l}0.026 \\
0.842\end{array}$ & $\begin{array}{l}0.137 \\
0.625\end{array}$ & $\begin{array}{l}0.021 \\
0.856\end{array}$ & $\begin{array}{l}0.013 \\
0.838\end{array}$ & $\begin{array}{l}0.098 \\
0.735\end{array}$ \\
\hline Between & 0.022 & 0.046 & 0.106 & 0.010 & 0.006 & 0.076 \\
\hline
\end{tabular}

Notas: errores estándar entre paréntesis. Significatividad al $10 \%, 5 \%$ y $1 \%$ : *,**,***, respectivamente.

Como se mencionó anteriormente, es lógico pensar que la diferencia en el comportamiento de la inscripción de los estudiantes de distintos grupos de edad está relacionada con los programas a los que se inscriben. Es mucho más probable que un estudiante menor de 24 años se encuentre estudiando su carrera de grado, o quizás una maestría, mientras que un estudiante mayor a 30 años probablemente estudie una maestría o un doctorado, y con menor probabilidad una carrera de grado. Por desgracia las bases de datos que contienen información por edad y programa son bastante incompletas y dificultan el estudio. Por esto se procedió a estimar el modelo por separado para los distintos programas sin distinción por edades. Otra falencia de la base es que 
no permite la desagregación entre programas de ciclo corto, grado y maestrías sino hasta el cambio en la clasificación ISCED realizado en 2011. De todos modos, estos programas tienen ciertas características en común y que los distinguen de programas de doctorado. Los primeros tienden a ser programas de menor duración, y muchas veces con un enfoque más profesional, mientras que los programas de doctorado suelen ser más largos y con enfoque académico. En la Tabla 4 pueden observarse los resultados de regresar el modelo por sexo según el programa terciario. Puede notarse que para los programas de ciclo corto/grado/maestría se reportan dos columnas. La primera de ellas estima el modelo utilizando como variable explicativa la tasa de desempleo de personas cuyo máximo nivel académico alcanzado es secundario superior/post-secundario no terciario, mientras que la segunda columna utiliza la tasa de desempleo de personas con estudios de nivel terciario.

Tabla 5: Inscripción universitaria por edad según nivel de PBI per cápita

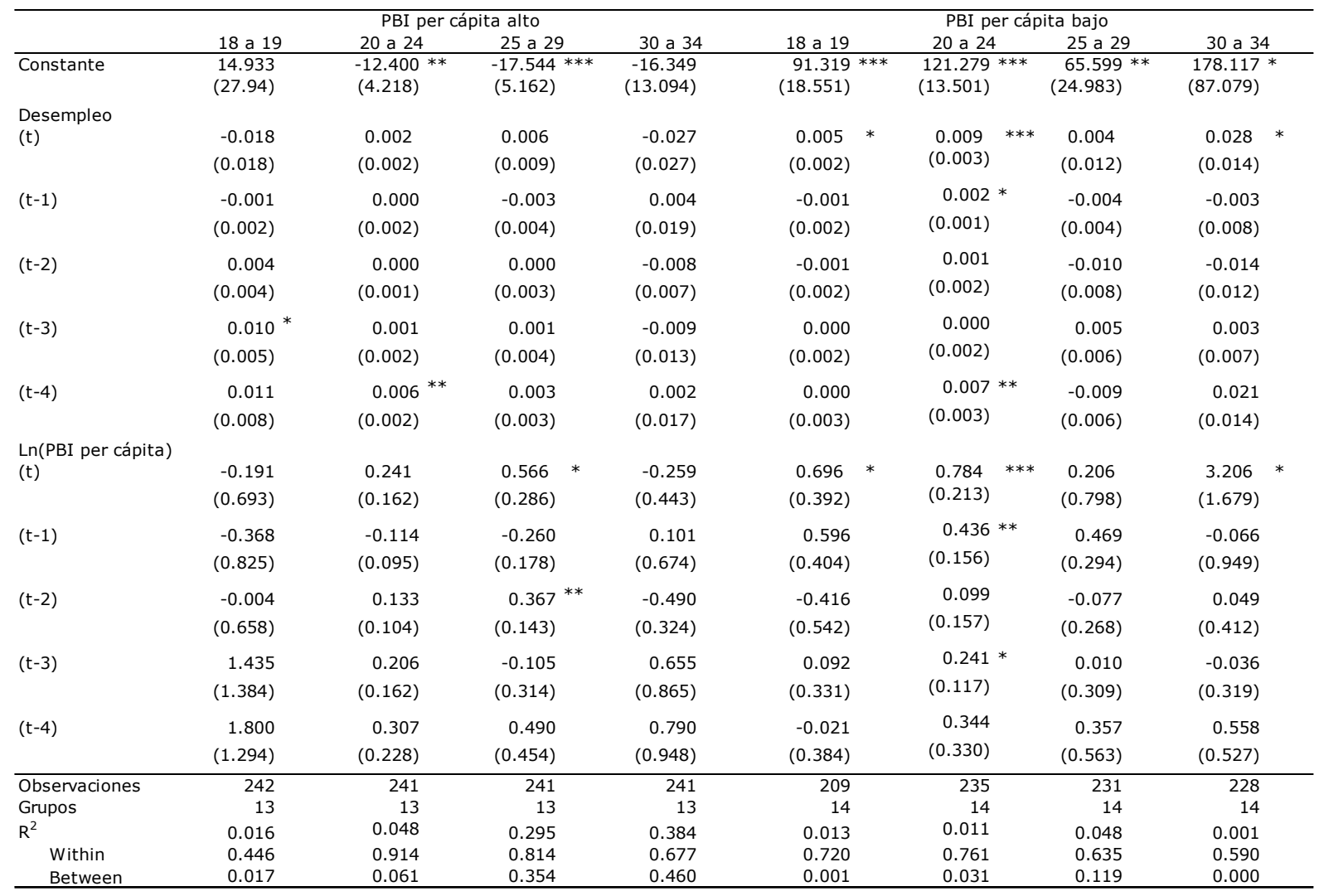

Notas: errores estándar entre paréntesis. Significatividad al $10 \%, 5 \%$ y $1 \%$ : *,**,***, respectivamente.

El estudio realizado por Bedard \& Herman (2008) encontró que la inscripción masculina en maestrías se comporta de manera pro-cíclica, en doctorados contra-cíclica y la inscripción femenina no se vería afectada por el ciclo económico. Sin embargo, no todos los resultados obtenidos se condicen con los de aquel trabajo. En primer lugar, tanto en la inscripción femenina como en la masculina y para ambas tasas de desempleo, se 
observan efectos significativos y positivos de ésta sobre la inscripción a programas de ciclo corto/grado/maestría. Además, nuevamente para ambos sexos, el PBI per cápita se asocia positivamente a la inscripción a este tipo de programas, no solo en el mismo período sino también en períodos subsiguientes por sus efectos rezagados. Incluso en la inscripción femenina el efecto pro-cíclico del PBI per cápita se observa mayor en magnitud que en la inscripción masculina. En cuanto a la inscripción a programas de doctorado se observan efectos significativos con rezagos de tres y cuatro años, lo cual podría interpretarse que se debe en realidad a los efectos directos del ciclo económico en programas de maestría, a los cuales los estudiantes de doctorado se habrían estado inscribiendo varios años antes.

Tabla 6: Inscripción por edad según nivel profundidad financiera

\begin{tabular}{|c|c|c|c|c|c|c|c|c|c|c|c|}
\hline & 18 a 19 & 20 a 24 & 25 a 29 & 30 a 34 & 18 a 19 & \multicolumn{2}{|l|}{20 a 24} & \multicolumn{2}{|c|}{25 a 29} & \multicolumn{2}{|l|}{30 a 34} \\
\hline Constante & $\begin{array}{r}-22.480 \\
(27.53)\end{array}$ & $\begin{array}{r}13.548 \\
(8.771)\end{array}$ & $\begin{array}{l}-29.786 \\
(20.07)\end{array}$ & $\begin{array}{r}-12.718 \\
(54.55)\end{array}$ & $\begin{array}{l}152.031 * * * \\
(42.24)\end{array}$ & $\begin{array}{c}104.327 \\
(9.935)\end{array}$ & $* * *$ & $\begin{array}{r}63.834 \\
(16.751)\end{array}$ & $* * *$ & $\begin{array}{l}140.980 \\
(35.757)\end{array}$ & $* * *$ \\
\hline $\begin{array}{l}\text { Desempleo } \\
(\mathrm{t})\end{array}$ & $\begin{array}{r}0.003 \\
(0.004)\end{array}$ & $\begin{array}{r}0.002 \\
(0.002)\end{array}$ & $\begin{array}{r}-0.006 \\
(0.006)\end{array}$ & $\begin{array}{r}-0.011 \\
(0.027)\end{array}$ & $\begin{array}{r}-0.009 \\
(0.010)\end{array}$ & $\begin{array}{r}0.008 \\
(0.002)\end{array}$ & $* * *$ & $\begin{array}{r}0.021 \\
(0.006)\end{array}$ & $* * *$ & $\begin{array}{r}0.024 \\
(0.012)\end{array}$ & * \\
\hline$(\mathrm{t}-1)$ & $\begin{array}{r}-0.004 \\
(0.003)\end{array}$ & $\begin{array}{r}0.001 \\
(0.001)\end{array}$ & $\begin{array}{r}-0.007 \\
(0.004)\end{array}$ & $\begin{array}{r}0.004 \\
(0.016)\end{array}$ & $\begin{array}{r}0.000 \\
(0.002)\end{array}$ & $\begin{array}{r}0.000 \\
(0.001)\end{array}$ & & $\begin{array}{r}0.001 \\
(0.004)\end{array}$ & & $\begin{array}{c}-0.007 \\
(0.010)\end{array}$ & \\
\hline$(t-2)$ & $\begin{array}{r}0.000 \\
(0.002)\end{array}$ & $\begin{array}{r}0.003 \\
(0.002)\end{array}$ & $\begin{array}{r}-0.011 \\
(0.007)\end{array}$ & $\begin{array}{r}-0.011 \\
(0.015)\end{array}$ & $\begin{array}{r}0.003 \\
(0.003)\end{array}$ & $\begin{array}{r}-0.001 \\
(0.001)\end{array}$ & & $\begin{array}{r}-0.002 \\
(0.004)\end{array}$ & & $\begin{array}{r}-0.014 \\
(0.007)\end{array}$ & * \\
\hline$(t-3)$ & $\begin{array}{r}0.004 \\
(0.005)\end{array}$ & $\begin{array}{r}0.001 \\
(0.003)\end{array}$ & $\begin{array}{r}0.002 \\
(0.004)\end{array}$ & $\begin{array}{r}-0.010 \\
(0.008)\end{array}$ & $\begin{array}{r}0.002 \\
(0.003)\end{array}$ & $\begin{array}{r}0.002 \\
(0.001)\end{array}$ & & $\begin{array}{r}-0.001 \\
(0.004)\end{array}$ & & $\begin{array}{r}0.005 \\
(0.008)\end{array}$ & \\
\hline$(t-4)$ & $\begin{array}{r}0.001 \\
(0.005)\end{array}$ & $\begin{array}{l}0.007 * * * \\
(0.002)\end{array}$ & $\begin{array}{r}0.003 \\
(0.005)\end{array}$ & $\begin{array}{r}0.004 \\
(0.019)\end{array}$ & $\begin{array}{r}0.002 \\
(0.005)\end{array}$ & $\begin{array}{r}0.004 * \\
(0.002)\end{array}$ & * & $\begin{array}{r}-0.001 \\
(0.004)\end{array}$ & & $\begin{array}{r}0.021 \\
(0.013)\end{array}$ & \\
\hline $\begin{array}{l}\text { Ln(PBI per cápita) } \\
\text { (t) }\end{array}$ & $\begin{array}{r}-0.179 \\
(0.609)\end{array}$ & $\begin{array}{r}0.385 \text { ** } \\
(0.146)\end{array}$ & $\begin{array}{r}-0.346 \\
(0.756)\end{array}$ & $\begin{array}{r}1.863 \\
(2.457)\end{array}$ & $\begin{array}{r}1.138 \\
(0.674)\end{array}$ & $\begin{array}{r}0.740 \\
(0.211)\end{array}$ & $* * *$ & $\begin{array}{r}0.992 \\
(0.379)\end{array}$ & ** & $\begin{array}{r}1.817 \\
(0.597)\end{array}$ & $* * *$ \\
\hline$(t-1)$ & $\begin{array}{r}0.545 \\
(0.360)\end{array}$ & $\begin{array}{r}-0.042 \\
(0.145)\end{array}$ & $\begin{array}{r}-0.346 \\
(0.756)\end{array}$ & $\begin{array}{r}-0.136 \\
(1.182)\end{array}$ & $\begin{array}{r}-0.358 \\
(0.617)\end{array}$ & $\begin{array}{r}0.339 * \\
(0.130)\end{array}$ & $* *$ & $\begin{array}{r}0.692 \\
(0.256)\end{array}$ & ** & $\begin{array}{r}0.352 \\
(0.476)\end{array}$ & \\
\hline$(t-2)$ & $\begin{array}{r}-0.269 \\
(0.556)\end{array}$ & $\begin{array}{r}0.238 \\
(0.148)\end{array}$ & $\begin{array}{r}0.223 \\
(0.293)\end{array}$ & $\begin{array}{c}-0.797 \\
(0.529)\end{array}$ & $\begin{array}{r}-0.042 \\
(0.485)\end{array}$ & $\begin{array}{r}0.211 * \\
(0.094)\end{array}$ & $* *$ & $\begin{array}{r}-0.166 \\
(0.234)\end{array}$ & & $\begin{array}{r}0.205 \\
(0.232)\end{array}$ & \\
\hline$(t-3)$ & $\begin{array}{r}0.039 \\
(0.504)\end{array}$ & $\begin{array}{r}0.347 \\
(0.205)\end{array}$ & $\begin{array}{l}-0.427 \\
(0.490)\end{array}$ & $\begin{array}{r}0.306 \\
(1.026)\end{array}$ & $\begin{array}{r}1.235 \\
(1.113)\end{array}$ & $\begin{array}{r}0.288 \\
(0.110)\end{array}$ & $* *$ & $\begin{array}{r}0.029 \\
(0.236)\end{array}$ & & $\begin{array}{r}0.116 \\
(0.266)\end{array}$ & \\
\hline$(t-4)$ & $\begin{array}{r}0.243 \\
(0.307) \\
\end{array}$ & $\begin{array}{r}0.090 \\
(0.181)\end{array}$ & $\begin{array}{c}0.996 * \\
(0.532)\end{array}$ & $\begin{array}{r}0.878 \\
(1.301) \\
\end{array}$ & $\begin{array}{r}0.494 \\
(0.499) \\
\end{array}$ & $\begin{array}{r}0.619 \\
(0.273) \\
\end{array}$ & $* *$ & $\begin{array}{r}-0.094 \\
(0.394) \\
\end{array}$ & & $\begin{array}{r}0.648 \\
(0.453) \\
\end{array}$ & \\
\hline Observaciones & 228 & 233 & 229 & 229 & 223 & 243 & & 243 & & 240 & \\
\hline Grupos & 13 & 13 & 13 & 13 & 14 & 14 & & 14 & & 14 & \\
\hline $\mathrm{R}^{2}$ & 0.042 & 0.001 & 0.179 & 0.032 & 0.098 & 0.140 & & 0.070 & & 0.062 & \\
\hline $\begin{array}{l}\text { Within } \\
\text { Between }\end{array}$ & $\begin{array}{l}0.689 \\
0.013\end{array}$ & $\begin{array}{l}0.874 \\
0.000\end{array}$ & $\begin{array}{l}0.684 \\
0.240 \\
\end{array}$ & $\begin{array}{l}0.574 \\
0.020 \\
\end{array}$ & $\begin{array}{l}0.404 \\
0.145 \\
\end{array}$ & $\begin{array}{l}0.857 \\
0.103\end{array}$ & & $\begin{array}{l}0.784 \\
0.034\end{array}$ & & $\begin{array}{l}0.689 \\
0.031\end{array}$ & \\
\hline
\end{tabular}

Notas: errores estándar entre paréntesis. Significatividad al $10 \%, 5 \%$ y $1 \%$ : *,**, ***, respectivamente.

Trabajos anteriores como Sakellaris \& Spilimbergo (2000) y Schafmeister (2016) examinan el comportamiento de la inscripción y los efectos del ciclo económico en ella separando los países en sub-grupos según criterios como la pertenencia a la OCDE, el nivel de ingresos, o el nivel de crédito al sector privado como porcentaje del PBI (como proxi para restricciones de liquidez). En la Tabla 5 se pueden encontrar los resultados de correr el modelo por separado para dos grupos de países según su PBI per cápita. La 
muestra se dividió según la mediana del PBI per cápita (en euros constantes de 2010) para el año 2002, primer año del que se tiene información para todos los países bajo estudio.

Una de las hipótesis planteadas al inicio del trabajo fue que el nivel de ingresos juega un rol en el efecto del ciclo económico sobre la inscripción universitaria. En base a los resultados de Sakellaris \& Spilimbergo (2000), se esperaba que un comportamiento contra-cíclico en países de ingresos altos y pro-cíclico en los países de menores ingresos, debido a las diferencias en la capacidad de ahorro. Sin embargo, esto no se comprueba en los resultados reportados. Observando la Tabla 5 es difícil poder afirmar que la inscripción en países de menores ingresos sigue un patrón marcadamente más procíclico que en países de mayores ingresos. En particular, para el grupo 25-29, no se observan efectos significativos del ciclo económico sobre la inscripción en países con menores niveles de ingresos. Por otro lado, en el grupo de PBI per cápita alto, la inscripción de este rango etario pareciera tener un comportamiento pro-cíclico, dado que un incremento de $1 \%$ en el PBI per cápita se asocia a un incremento en la inscripción de $0,57 \%$ en el mismo período, y de $0,37 \%$ con dos períodos de rezago. Cabe mencionar que para la sub-muestra de países de menores niveles de ingreso se observan efectos contra-cíclicos de la tasa de desempleo en la inscripción para todos los rangos de edades analizados a excepción de 25-29. Un incremento de 1 punto porcentual en la tasa de desempleo se asocia a incrementos de $0,49 \%, 0,87 \%$ y $2,79 \%$ en la inscripción de los grupos 18-19, 20-24 y 30-34, respectivamente. Aunque también se observan importantes efectos pro-cíclicos del PBI per cápita en la inscripción en este grupo de países.

Otra de las hipótesis planteadas al inicio del trabajo era que también el grado de profundidad financiera afectaba a la relación entre inscripción universitaria y ciclo económico, argumentando que el sistema financiero podría proveer liquidez ante la caída de los ingresos reduciendo la pro-ciclicidad de la inscripción. Los resultados reportados en la Tabla 5 confirman parcialmente esta hipótesis. Para dividir la muestra se utilizó el dato de crédito doméstico al sector privado como porcentaje del PBI que surge de las bases de datos del Banco Mundial para el año 2010 (primer año del que se tiene registro para todos los países de la muestra) utilizando la mediana como valor de corte. La Tabla 6 muestra que los efectos del ciclo económico en la inscripción a programas de educación superior son mayores en países con menores niveles de crédito doméstico. Sin embargo, no solo se observan mayores efectos pro-cíclicos del PBI per cápita, sino también mayores efectos contra-cíclicos de la tasa de desempleo.

\section{Conclusiones}

El objetivo del trabajo fue avanzar y aportar en la comprensión del efecto del ciclo económico en la acumulación de capital humano. Para esto se aprovecharon las bases de datos de Eurostat que proveen información relevante para una gran cantidad de países y un período de tiempo suficientemente extenso. Se pusieron a prueba diferentes hipótesis que surgen de los resultados de trabajos anteriores y se alcanzaron resultados que, si bien resuelven algunas de las preguntas, dejan planteadas otras. Se comenzó 
estudiando los efectos del desempleo y el PBI per cápita en la inscripción universitaria para diferentes grupos sociodemográficos, para, en una segunda instancia, analizar dichos efectos en la inscripción a distintos programas de nivel terciario. Finalmente se procedió a estudiar el comportamiento de la inscripción para diferentes sub-muestras, partiendo en dos la muestra inicial de 27 países primero según sus niveles de PBI per cápita y luego según su nivel de profundidad financiera.

En primer lugar, se encontró que la inscripción universitaria se ve afectada por el ciclo económico. Éste es el principal resultado del trabajo. El efecto, sin embargo, se observa únicamente en estudiantes de entre 20 y 24 años, donde se pueden observar tanto los efectos contra-cíclicos relacionados al costo de oportunidad como los efectos pro-cíclicos del PBI per cápita. La presencia de ambos efectos posiblemente se deba a la existencia de grupos diferenciados que se ven afectados por cada uno. También se observa que el ciclo afecta de forma diferenciada a la inscripción masculina de la femenina. En particular, la inscripción de mujeres de entre 25 y 29 años parece verse considerablemente más afectada por cambios en la tasa de desempleo que la masculina para el mismo, o cualquier otro rango de edades, lo cual puede interpretarse como una mayor sensibilidad a variaciones en los costos de oportunidad de la inversión en capital humano. Por su parte, en la inscripción femenina del grupo 30-34 sobresale el efecto contemporáneo del PBI per cápita en la inscripción, evidenciando un comportamiento marcadamente pro-cíclico que no se observa en la inscripción masculina.

En segundo lugar, se encuentran también efectos diferenciados del ciclo económico en la inscripción a distintos programas. Para ambos sexos se encuentran efectos significativos y positivos tanto del desempleo como del PBI per cápita en la inscripción a programas de ciclo corto/grado/maestría, siendo los efectos de mayor magnitud en la inscripción femenina, lo cual difiere de resultados de trabajos anteriores. Los efectos del ciclo económico parecen de menor magnitud en la inscripción a programas de doctorado.

En tercer lugar, se comprueba la hipótesis de un comportamiento diferenciado de la inscripción en países de ingresos altos respecto de países de ingresos bajos. Sin embargo, los resultados no son tan claros como se esperaban. No se puede afirmar que la inscripción en países de menores ingresos sigue un patrón marcadamente más procíclico que en países de mayores ingresos. De hecho, para el rango 25-29, no se observan efectos del ciclo económico en la inscripción de países de menores ingresos, pero si en países de mayores ingresos, donde la inscripción sigue para ese rango de edades un comportamiento pro-cíclico. Para los restantes rangos etarios, los efectos tanto pro-cíclicos como contra-cíclicos parecen mayores en países cuyo PBI es más bajo.

Por último, se comprueba sólo parcialmente la hipótesis acerca del rol de los sistemas financieros como proveedores de liquidez que permitirían un comportamiento menos pro-cíclico y más contra-cíclico de la inscripción. Si bien se observa que en países de mayor profundidad financiera la inscripción se ve menos afectada por fluctuaciones en el PBI per cápita, también puede observarse un menor efecto de la tasa de desempleo. Por tanto, no se puede afirmar que en países con mayores niveles de crédito doméstico la inscripción sea más contra-cíclica, solamente que es menos cíclica. 
Si bien, como se mencionó anteriormente, el estudio en base a un panel de datos permitió aportar nueva información al debate acerca de la relación entre la acumulación de capital humano y el ciclo económico, la metodología escogida no deja de presentar limitaciones. En primer lugar, al no disponer en las bases de datos de Eurostat de series de tiempo por país relacionadas a variables como los créditos estudiantiles, costos directos de estudiar, o asistencia financiera de los gobiernos a los estudiantes, las conclusiones alcanzadas solo pueden ser parciales. Esto se debe a que dichas variables afectan directamente a la acumulación de capital humano y probablemente fluctúen a lo largo del ciclo económico. Otra importante limitación relacionada a la metodología escogida es que la utilización de datos a nivel agregado no permite analizar las restricciones presupuestarias individuales que influyen en las decisiones de inversión en capital humano.

El estudio realizado permite observar la complejidad de la relación entre el ciclo económico y la acumulación de capital humano. Sin embargo, la complejidad es tal que para lograr comprenderla serían necesarios estudios que profundicen en la caracterización de los estudiantes de los distintos países y en su toma de decisiones mediante la utilización de datos a nivel individuos. Solo comprendiendo correctamente la toma de decisiones de los estudiantes pueden implementarse políticas que favorezcan a la acumulación de capital humano, y así contribuir a una sociedad más educada, y con menores niveles de pobreza y desigualdad. 


\section{Referencias}

Barakat, B., Holler, J., Prettner, K., \& Schuster, J. (2010). The impact of the economic crisis on labour and education in Europe. Vienna Institute of Demography Working Papers No. 1006. Disponible en: https://EconPapers.repec.org/RePEc:vid:wpaper:1006

Becker, G. S. (1964). Human capital: A theoretical and empirical analysis, with special reference to education. University of Chicago Press.

Bedard, K., \& Herman, D. A. (2008). Who goes to graduate/professional school? The importance of economic fluctuations, undergraduate field, and ability. Economics of Education Review, 27(2), 197-210.

Bell, D., \& Blanchflower, D. G. (2010). Young people and recession: A lost generation? Paper presented at the fifty-second panel meeting of Economic Policy, October 22-23, 2010.

Betts, J. R., \& McFarland, L. L. (1995). Safe port in a storm: The impact of labor market conditions on community college enrollments. Journal of Human Resources, 30(4), 741765. https://doi.org/10.2307/146230

Bogan, V.L., \& Wu, D. (2018). Business cycles, race, and investment in graduate education. Journal of Economics, Race and Policy, 1, 142-175 https://doi.org/10.1007/s41996-018-0004-x

Cochrane, J. H. (1994). Permanent and transitory components of GNP and stock prices. The Quarterly Journal of Economics, 109(1), 241-265.

Dellas, H., \& Sakellaris, P. (2003). On the cyclicality of schooling: theory and evidence. Oxford Economic Papers, 55(1), 148-172. https://doi.org/10.1093/oep/55.1.148

Drozdowicz-Bieć, M. (2011). Reasons why Poland avoided the 2007-2009 recession. Prace I Materiały, 86(2), 39-66.

Disponible en: https://EconPapers.repec.org/RePEc:wsd:irgpim:v:86:y:2011:i:2:p:3966

Flug, K., Spilimbergo, A., \& Wachtenheim, E. (1998). Investment in education: do economic volatility and credit constraints matter? Journal of Development Economics, 55(2), 465-481. https://doi.org/10.1016/S0304-3878(98)00045-5

Foote, A., \& Grosz, M. (2020). The effect of local labor market downturns on postsecondary enrollment and program choice. Education Finance and Policy, 15(4), 593-622. https://doi.org/10.1162/edfp_a_00288

Ghita, S., Titan, E., \& Boboc, C. (2013). How does the economic-financial crisis affect our education? Study on EU-28 Countries. Ovidius University Annals, Series Economic Sciences, 13(2), 112-117.

Disponible en: https://EconPapers.repec.org/RePEc:ovi:oviste:v:xiii:y:2013:i:2:p:112117

Goh, W. (2009). Is enrollment into graduate school affected by the business cycle? Stanford University, Department of Economics. 
Kärkkäinen, K. (2010). Summary of the June 2009 educationtoday Crisis Survey. OECD Education Working Papers No 43. https://doi.org/10.1787/5kmmsxbws77d-en

OCDE (2012). How has the global economic crisis affected people with different levels of education? Education Indicators in Focus.

Disponible en: http://www.oecd.org/dataoecd/35/44/49471658.pdf

Sakellaris, P., \& Spilimbergo, A. (2000). Business cycles and investment in human capital: international evidence on higher education. Carnegie-Rochester Conference Series on Public Policy, 52, 221-256. https://doi.org/10.1016/S0167-2231(00)00024-5

Schafmeister, F. (2016). The Cyclicality of Enrollment in Tertiary Education [Tesis de maestría, Erasmus Universiteit Rotterdam].

Disponible en: https://thesis.eur.nl/pub/35059/Schafmeister.pdf

Skrbinjek, V., Lesjak, D., \& Šušteršič, J. (2015). Impact of economic crisis on student demand for tertiary education. In Managing intellectual capital and innovation for sustainable and inclusive society: Proceedings of the MakeLearn and TIIM Joint International Conference (pp. 2267-2274). 27-29 May 2015, Bari: ToKnowPress. Disponible en: https://EconPapers.repec.org/RePEc:tkp:mklp15:2267-2274

Van Damme, D., \& Kärkkäinen, K. (2011). OECD Educationtoday Crisis Survey 2010. OECD Education Working Papers No 56. https://doi.org/10.1787/5kgj1r9zk09x-en

Varghese, N. V. (2010). Running to stand still: Higher education in a period of global economic crisis. Research Papers IIEP. 